\title{
Relative Haag Duality for the Free Field in Fock Representation
}

\author{
Paolo Camassa* \\ Institut für Theoretische Physik, Georg-August Universität Göttingen \\ Friedrich-Hund-Platz, 1 - 37077 - Göttingen - Germany \\ paolo.camassa@istruzione.it
}

September 27, 2018

\begin{abstract}
We consider a natural generalization of Haag duality to the case in which the observable algebra is restricted to a subset of the space-time and is not irreducible: the commutant and the causal complement have to be considered relatively to the ambient space. We prove this relative form of Haag duality under quite general conditions for the free scalar and electromagnetic field of space dimension $d \geq 2$ in the vacuum representation. Such property is interesting in view of a theory of superselection sectors for the electromagnetic field.
\end{abstract}

\section{Introduction and Motivations}

In the framework of algebraic quantum field theory, the property of Haag duality [17, which is a maximality condition with respect to the locality principle, has proven to be a powerful tool especially for the theory of superselection sectors. It has been exploited in the DHR theory [12, 13, 14, 15, where superselection sectors are classified as unitary equivalence classes of representations of the observable algebra satisfying a selection criterion. In the presence of long range interactions, like the electromagnetic field, the DHR criterion does not apply and the problem of how to distinguish the physical charges in the continuous infinity of inequivalent representations has to be solved. According to a proposal of Buchholz [8], the electric charge should be recognizable when one looks at the restriction of the representation of the observable algebra to a forward light cone and considers unitary equivalence classes of the representations restricted to any such cone.

From this point of view it is interesting to prove that the naturally generalized version of Haag duality holds when the ambient space-time is the forward light cone $V_{+}$. As a step in this direction, we consider here the free scalar and electromagnetic field on Minkowski space-time $\mathbb{R}^{d+1}$ in the representation of the vacuum state, with the corresponding net of von Neumann algebras $O \mapsto \mathfrak{R}(O)$, and restrict our attention to a globally hyperbolic subset $M \subset \mathbb{R}^{d+1}$ (as a special case $M=V_{+}$). Haag duality for double cone regions in $\mathbb{R}^{d+1}$ is well known 1, 2, 9, 25, 16, 22, 20, 18, 4. We prove here its relative form, where the commutant is replaced by the relative commutant with respect to the algebra $\mathfrak{R}(M)$ and the relative causal complement of a double cone is its causal complement inside $M$.

The technique used is essentially the following. From a general theorem on Fock representation of the abstract algebra of canonical commutation relations (CCR-algebra), recalled in Section 2] we know that Haag duality is equivalent to a duality property of the one-particle

*Supported by the EU network "Quantum Spaces - Noncommutative Geometry" HPRN-CT-2002-00280 
space. This one-particle space duality is generally proven using a Cauchy surface and the correspondence of solutions of the Klein-Gordon equations with their Cauchy data. In Section 3, we consider a globally hyperbolic space-time $M$, i.e. a space-time with a Cauchy (smooth) surface $\boldsymbol{\Sigma}$ : e.g. $M$ can be $V_{+}$, a double cone or a wedge region. We then consider the known structure of the one-particle space associated to such a generic surface $\boldsymbol{\Sigma}$. In Section 4, for the scalar free field of any mass and space-dimension $d \geq 2$, we first prove relative duality for a double cone $O$ and then for its relative commutant $O^{c}$, Theorem 4.8 Notice that, unlike the standard case, here duality for $O$ does not imply directly duality for $O^{c}$. In Section 5, we consider the electromagnetic free field and prove duality for a double cone $O$, Theorem [5.4 In view of a theory of superselection sectors for the electric charge, which can not be localized in a double cone, one expects that a charged state can be seen in the vacuum sector as the limit of states in which a charge is fixed and an opposite charge is moved to space-like infinity. Staying inside the forward light cone, the compensating charge can be moved along a trajectory which, approaching the surface of the cone, goes to light-like infinity with respect to the vertex of the cone and space-like infinity with respect to any internal point of the cone. To be more explicit, the compensating charge can be moved on a hyperboloid along a specific direction and the charged state obtained by this limit would be localized in a causally complete open region containing the trajectory. It is useful, therefore, to prove relative Haag duality for such a class of regions; this is the content of Theorem 5.14

\section{Fock Representation}

\subsection{Net of von Neumann Algebras in Fock Representation}

The Weyl CCR-algebra $\mathfrak{A}(H, \sigma)$ is well defined as an abstract $\mathrm{C}^{*}$-algebra canonically associated to the symplectic space $(H, \sigma)[24,6]$. Whenever we have a representation, or a state $\omega$ and the corresponding GNS representation $\pi_{\omega}$, we can also define von Neumann algebras by closing $\mathfrak{A}(H, \sigma)$ and its $\mathrm{C}^{*}$-subalgebras with respect to the weak topology.

When $H_{0}$ is a complex Hilbert space and the symplectic form $\sigma$ is the imaginary part of the scalar product, we have the Fock representation of $\mathfrak{A}\left(H_{0}, \sigma\right)$ and, closing the local algebras $\mathfrak{A}(V)$, for any subspace $V$ of $H_{0}$, with respect to the weak topology, we define a net of von Neumann algebras:

$$
\operatorname{subsp}\left(H_{0}\right) \ni V \mapsto \mathfrak{R}(V):=\pi_{0}(\mathfrak{A}(V))^{\prime \prime} \subset \mathcal{B}\left(\Gamma\left(H_{0}\right)\right),
$$

where $\operatorname{subsp}\left(H_{0}\right)$ indicates the set of closed real subspaces of $H_{0}$ and $\mathcal{B}\left(\Gamma\left(H_{0}\right)\right)$ is the algebra of bounded operators on the Fock space $\Gamma\left(H_{0}\right)$. We can actually define $\mathfrak{R}(V)$ for any $V$, even not closed, but it coincides with the algebra associated to its closure: $\mathfrak{R}(V)=\mathfrak{R}(\bar{V})$ (see e.g. [1] or [22]).

The set of closed subspaces is a complemented lattice, namely there is an order relation $\subset$ by inclusion, with respect to which the operations of supremum $\vee$ and infimum $\wedge$ are well defined, and an operation of symplectic complement '. The same is true for the set of von Neumann algebras in $\mathcal{B}\left(\Gamma\left(H_{0}\right)\right)$, where the complement is defined as the commutant. Notice that the complement is a closed object in both cases, w.r.t. the respective topology.

The map in 1 is a homomorphism of complemented lattices, emphasized by the use of the same notations for the lattice operations in both cases. This is the content of the following:

Theorem 2.1. (Araki) Let $H_{0}$ be a complex Hilbert space, $V, W, V_{\alpha} \in \operatorname{subsp}\left(H_{0}\right)$ (closed real subspaces of $\left.H_{0}\right)$, then

$$
\begin{aligned}
& \text { 1. } \mathfrak{R}(V) \supset \mathfrak{R}(W) \Longleftrightarrow V \supset W \text { and } \mathfrak{R}(V)=\mathfrak{R}(W) \Longleftrightarrow V=W \text { (isotony), } \\
& \text { 2. } \vee_{\alpha} \mathfrak{R}\left(V_{\alpha}\right)=\mathfrak{R}\left(\vee_{\alpha} V_{\alpha}\right) \text { (additivity), }
\end{aligned}
$$


3. $\wedge_{\alpha} \mathfrak{R}\left(V_{\alpha}\right)=\mathfrak{R}\left(\wedge_{\alpha} V_{\alpha}\right)$,

4. $\mathfrak{R}(V)^{\prime}=\mathfrak{R}\left(V^{\prime}\right)$ (abstract duality).

It was originally proven in [1], Th. 1; see also [22, 3].

Property 4 states that the commutant of the algebra associated to a subspace is exactly the algebra associated to the symplectic complement of that space. In [22] it is called abstract Haag duality. We generalize now this concept of duality to the context of a reducible net of von Neumann algebras, when the one-particle space is a real subspace of a complex Hilbert space $H_{0}$.

Definition 2.2. Let $H$ be a real symplectic topological space and $\operatorname{subsp}(H) \ni V \mapsto \mathfrak{R}(V) a$ net of von Neumann algebras. For any $V \in \operatorname{subsp}(H)$, let

$$
V^{c}:=\{f \in H: \sigma(f, g)=0 \quad \forall g \in V\}
$$

be the symplectic complement in the ambient space $H$; for any $\mathfrak{R}(V)$, let

$$
\mathfrak{R}(V)^{c}:=\{A \in \mathfrak{R}(H):[A, B]=0 \quad \forall B \in \mathfrak{R}(V)\}
$$

be the relative commutant in the ambient von Neumann algebra $\mathfrak{R}(H)$.

Note that the notations $V^{\prime}$ and $\mathfrak{R}(V)^{\prime}$ were used to indicate the same complements in the special case in which $H=H_{0}$ is a complex Hilbert space and $\mathfrak{R}(H)$ is the whole algebra of bounded operators on the representation space; thus the 1-complement is an involutive operation, although the $c$-complement is not in general.

Definition 2.3. A (not necessarily irreducible) net of represented von Neumann algebras $\operatorname{subsp}(H) \ni V \mapsto \mathfrak{R}(V)$ is said to satisfy abstract Haag duality iff, for every $V \in \operatorname{subsp}(H)$,

$$
\mathfrak{R}(V)^{c}=\mathfrak{R}\left(V^{c}\right)
$$

Let now choose a closed real subspace $H \subset H_{0}$ ( $H$ is not supposed to be invariant under the multiplication by $i$ ) such that $\sigma$ is still non-degenerate when restricted to $H$; it inherits the structure of a real Hilbert and symplectic space and the net in eq. 1 can be restricted to the net of von Neumann algebras $\operatorname{subsp}(H) \ni V \mapsto \mathfrak{R}(V)$ on the same representation space, which is in general no more irreducible. In that case one can write $V^{c}=V^{\prime} \cap H$ and $\mathfrak{R}(V)^{c}=\mathfrak{R}(V)^{\prime} \cap \mathfrak{R}(H)$.

Proposition 2.4. Let $H$ be a closed real subspace of a complex Hilbert space $H_{0}$, then the net of von Neumann algebras subsp $(H) \ni V \mapsto \mathfrak{R}(V)$ satisfies isotony, additivity and abstract Haag duality (Th. 2.1 and Def. [2.3).

Proof. Isotony and additivity are immediate consequences of the definition. Concerning abstract Haag duality, one checks that

$$
\mathfrak{R}(V)^{c}:=\mathfrak{R}(V)^{\prime} \cap \mathfrak{R}(H)=\mathfrak{R}\left(V^{\prime}\right) \cap \mathfrak{R}(H)=\mathfrak{R}\left(V^{\prime} \cap H\right)=\mathfrak{R}\left(V^{c}\right),
$$

applying properties 3 and 4 of Th. 2.1

\section{Geometric Structure of the KG Equation}

Let $M \subset \mathbb{R}^{d+1}$ be an open globally hyperbolic submanifold of Minkowski space-time, maximal in the sense that there is no bigger submanifold with the same Cauchy surfaces; examples are the forward light cone $V_{+}$, a double cone or $\mathbb{R}^{d+1}$ itself. Global hyperbolicity is used to define a natural symplectic structure on $C_{c}^{\infty}(M)$, corresponding to the commutator of free fields, whilst translations in Minkowski space-time are used to define an inner product, corresponding to the vacuum state (unique translation invariant Fock state). 


\subsection{Causal Structure in Minkowski Space-Time}

Minkowski space time has a natural causal structure:

Definition 3.1. For every subset $O \subset \mathbb{R}^{d+1}$ we define its causal complement as

$$
O^{\prime}:=\left\{x \in \mathbb{R}^{d+1}:(x-y)^{2}<0 \quad \forall y \in O\right\}
$$

where $x^{2}<0$ iff $x$ is spacelike. The causal completion of $O$ is $O^{\prime \prime}$.

As global hyperbolicity has a crucial role in the following, we will consider the family of regions that are causal completions of open subsets of a Cauchy surface for $M$. Let $\boldsymbol{\Sigma}$ be a Cauchy surface, $\mathbf{B} \subset \boldsymbol{\Sigma}$, the diamond $C(\mathbf{B})$ with base $\mathbf{B}$, defined as the set of points for which any non space-like straight line intersects $\mathbf{B}$, coincides with the causal completion of B: $C(\mathbf{B})=\mathbf{B}^{\prime \prime} . C(\mathbf{B})$ is the biggest region of space-time whose observables are completely determined by those of $\mathbf{B}$; the maximality of $M$ assumed above ensures that $C(\mathbf{B}) \subset M$. In order to define spaces of smooth functions on these regions we will consider open sets: it can be easily proven that int $(C(\mathbf{B}))=C(\operatorname{int}(\mathbf{B}))$ and therefore $C(\mathbf{B})$ is open when $\mathbf{B}$ is.

Definition 3.2. For every open subset $O \subset M$ we define $O^{c}$ as the interior of its (relative) causal complement in the ambient manifold $M$, i.e.

$$
O^{c}=\operatorname{int}\left\{x \in M:(x-y)^{2}<0 \quad \forall y \in O\right\}
$$

clearly, $O^{c}=\operatorname{int}\left(O^{\prime} \cap M\right)$.

It follows easily form the definition that, using a Cauchy surface, the causal complement is essentially reduced to set complement: for every open subset $\mathbf{B} \subset \boldsymbol{\Sigma}, C(\mathbf{B})^{c} \cap \boldsymbol{\Sigma}=\boldsymbol{\Sigma} \backslash \overline{\mathbf{B}}$ and hence

$$
C(\mathbf{B})^{c}=C(\boldsymbol{\Sigma} \backslash \overline{\mathbf{B}})
$$

\subsection{Symplectic Space on Globally Hyperbolic Space-Times}

Following [10, as $M$ is a globally hyperbolic $d+1$-dimensional time-oriented space-time, there exist two unique continuous operators $E_{+}, E_{-}$(whose integral kernels are the advanced and retarded Green functions) satisfying the following properties:

$$
\begin{gathered}
E_{ \pm}: C_{c}^{\infty}(M) \rightarrow C^{\infty}(M), \\
\left(\square+m^{2}\right) E_{ \pm}=E_{ \pm}\left(\square+m^{2}\right)=\mathbf{1}_{C_{c}^{\infty}(M)}, \\
\operatorname{supp}\left(E_{ \pm} f\right) \subset J_{ \pm}(\operatorname{supp} f),
\end{gathered}
$$

where $J_{ \pm}(O)$ is the set of all points that can be reached from a point of the set $O$ with a forward/backward time-like or light-like line; on Minkowski space-time their Fourier transforms are $\widehat{E_{ \pm}}(p)=\frac{1}{p^{2}-m^{2} \pm i p_{0} \epsilon}$. We can then define the propagator

$$
E:=E_{+}-E_{-}: C_{c}^{\infty}(M) \rightarrow C^{\infty}(M),
$$

which satisfies the Klein-Gordon equation and whose support is in the time-like cone: $\operatorname{supp}(E f) \subset J_{+}(\operatorname{supp} f) \cup J_{-}(\operatorname{supp} f)$. Its Fourier transform is $\widehat{E}(p)=-i \epsilon\left(p_{0}\right) \delta\left(p^{2}-m^{2}\right)$, where $\epsilon\left(p_{0}\right)$ is the signum of $p_{0}$. The transposes are $E_{ \pm}^{\prime}=E_{\mp}: C_{c}^{-\infty}(M) \rightarrow C^{-\infty}(M)$, indicating with the same symbol the extensions to the dual spaces, and consequently $E^{\prime}=-E$.

For any choice of a Cauchy surface $\boldsymbol{\Sigma}$, we have the following continuous maps which associate to a solution of the Klein-Gordon equation its Cauchy data:

$$
\begin{array}{cccc}
\rho_{0}, \rho_{1}: C_{c}^{\infty}(M) & \rightarrow & C_{c}^{\infty}(\boldsymbol{\Sigma}) \\
f & \mapsto \quad \rho_{0} f:=\left.f\right|_{\boldsymbol{\Sigma}}, \rho_{1} f:=\left.\frac{\partial}{\partial n} f\right|_{\boldsymbol{\Sigma}},
\end{array}
$$


where $\frac{\partial}{\partial n}$ denotes the normal derivative w.r.t. $\boldsymbol{\Sigma}$. The dual operators are $\rho_{0}^{\prime}, \rho_{1}^{\prime}: C^{-\infty}(\boldsymbol{\Sigma}) \rightarrow$ $C^{-\infty}(M)$. We have the following equality of maps from $C_{c}^{\infty}(M)$ to $C^{\infty}(M)$ (see [10]):

$$
E=E\left(\rho_{0}^{\prime} \rho_{1}-\rho_{1}^{\prime} \rho_{0}\right) E .
$$

Defining then, for $i, j \in \mathbb{Z}_{2}, E_{i j}:=\rho_{i+1} E \rho_{j+1}^{\prime}: C_{c}^{\infty}(\boldsymbol{\Sigma}) \rightarrow C_{c}^{\infty}(\boldsymbol{\Sigma})$, we have

$$
E_{00}=E_{11}=\mathbf{0}, \quad E_{01}=-E_{10}=\mathbf{1}_{C_{c}^{\infty}(\boldsymbol{\Sigma})} .
$$

On the space $C_{c}^{\infty}(M)$, a natural bilinear antisymmetric form is defined by $\sigma(f, g):=$ $\langle f, E g\rangle$, where $\langle\cdot, \cdot\rangle$ denotes the canonical $L^{2}$ scalar product or, alternatively, the action of a function as a distribution on another function. One can easily prove that it is non-degenerate on the quotient space $\frac{C_{c}^{\infty}(M)}{\sim}$, where $f \sim g$ iff $E f=E g$. It defines therefore a symplectic form:

$$
\begin{array}{rlll}
\sigma: \frac{C_{c}^{\infty}(M)}{[\tilde{f}]} \times \frac{C_{c}^{\infty}(M)}{\tilde{\sigma}]} & \rightarrow & \mathbb{R} \\
& \mapsto \sigma([f],[g]):=\langle f, E g\rangle
\end{array} .
$$

The obvious isomorphic correspondence between equivalence classes $[f]$ and solutions $E f$ of the Klein-Gordon equation can be pushed, given any Cauchy surface $\boldsymbol{\Sigma}$, to the Cauchy data. To every $E f$ are uniquely associated its Cauchy data $f_{0}=\rho_{0} E f, f_{1}=\rho_{1} E f$ on $\boldsymbol{\Sigma}$. In the other direction, for any couple of functions $f_{0}, f_{1} \in C_{c}^{\infty}(\boldsymbol{\Sigma})$, there exists a function $f \in C_{c}^{\infty}(M)$ such that $\rho_{i} E f=f_{i}, i \in \mathbb{Z}_{2}$ (see again [10]). We have thus an isomorphism

$$
\frac{C_{c}^{\infty}(M)}{\sim} \simeq C_{c}^{\infty}(\boldsymbol{\Sigma}) \oplus C_{c}^{\infty}(\boldsymbol{\Sigma})
$$

and, in terms of the Cauchy data, using 6 and 7 the symplectic form takes the simplest form:

$$
\sigma\left(f_{0} \oplus f_{1}, g_{0} \oplus g_{1}\right)=\left\langle f_{0}, g_{1}\right\rangle-\left\langle f_{1}, g_{0}\right\rangle .
$$

We can summarize the material in this subsection in the following

Proposition 3.3. To a time oriented globally hyperbolic space-time $M$ is associated the symplectic space $\frac{C_{c}^{\infty}(M)}{\sim}$ of solutions of the Klein-Gordon equation, for any mass $m$, with symplectic form 8 . To any Cauchy surface $\boldsymbol{\Sigma}$ is associated the symplectic space of Cauchy data $C_{c}^{\infty}(\boldsymbol{\Sigma}) \oplus C_{c}^{\infty}(\boldsymbol{\Sigma})$ with symplectic form 10 . They are isomorphic via $\mathbf{0}$.

\subsection{One-Particle Hilbert Space}

Let us now consider the embedding of $M$ in Minkowski space-time, where we can use spacetime translations and Fourier transform.

We can define in a natural way a complex structure on $H_{0}:=H\left(\mathbb{R}^{d+1}\right)$, i.e. an operator $J$ which is a symplectic square root of $\mathbf{- 1}$ given by multiplication in Fourier transform by $i \epsilon\left(p_{0}\right): \widehat{J f}(p):=i \epsilon\left(p_{0}\right) \widehat{f}(p)$ (notice that multiplication by $i$ does not preserve the space of Fourier transforms of real functions). As $J$ is not a local operator, one cannot expect that $H(M)$ is invariant under $J$, unless $H(M)=H_{0}$. The propagator $E: C_{c}^{\infty}\left(\mathbb{R}^{d+1}\right) \rightarrow$ $C^{\infty}\left(\mathbb{R}^{d+1}\right)$ in Minkowski space-time, when restricted to $M$, is exactly the operator in eq. 4. as it satisfies the required properties. The operator

$$
P=J E: C_{c}^{\infty}\left(\mathbb{R}^{d+1}\right) \rightarrow C^{\infty}\left(\mathbb{R}^{d+1}\right)
$$

is positive as a quadratic form and is associated to the vacuum state $\omega$ in the following way. On $C_{c}^{\infty}\left(\mathbb{R}^{d+1}\right) / \sim$, the bilinear form $(f, g)_{\mathbb{R}}:=\langle f, P g\rangle$ is a real scalar product and

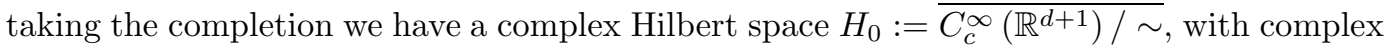
structure given by $J$. The symplectic form $\sigma(f, g)=\langle f, E g\rangle$ can be extended to $H_{0}$ and 
there is a Fock state $\omega$ on the CCR-algebra $\mathfrak{A}\left(H_{0}, \sigma\right)$ whose two point function is a complex scalar product

$$
\omega(f, g)=(f, g)_{\mathbb{R}}+i \sigma(f, g) .
$$

Let $\boldsymbol{\Sigma} \subset M \subset \mathbb{R}^{d+1}$ be a Cauchy surface for $M$ and $\rho_{i}$ the corresponding operators (cf. [5). From the relation $P=J E$, restricted to $M$, and 6 it follows that

$$
P=P\left(\rho_{0}^{\prime} \rho_{1}-\rho_{1}^{\prime} \rho_{0}\right) E \quad \text { on } C_{c}^{\infty}(M) .
$$

Using this formula, one verifies that, in terms of the Cauchy data, $P$ has the form

$$
\langle f, P g\rangle=\left\langle f_{1}, P_{11} g_{1}\right\rangle+\left\langle f_{0}, P_{00} g_{0}\right\rangle-\left\langle f_{0}, P_{01} g_{1}\right\rangle-\left\langle f_{1}, P_{10} g_{0}\right\rangle,
$$

where, for $i, j \in \mathbb{Z}_{2}, P_{i j}:=\rho_{i+1} P \rho_{j+1}^{\prime}: C_{c}^{\infty}(\boldsymbol{\Sigma}) \rightarrow C^{\infty}(\boldsymbol{\Sigma})$.

The space-time was assumed to be time-oriented. Time orientation enters in the definition of $\sigma$ through the operator $E$ : if we change the time orientation, then $E \mapsto-E, \sigma \mapsto-\sigma$, the isomorphism in 9 is replaced by $\rho_{0} \mapsto \rho_{0}, \rho_{1} \mapsto-\rho_{1}$ and therefore

$$
f_{0} \oplus f_{1} \mapsto f_{0} \oplus-f_{1} .
$$

Under time orientation inversion $J \mapsto-J$ and $P$ is invariant; it follows that 14 must be invariant under the map [15] and thus

$$
P_{10}=P_{01}=0 .
$$

As a consequence, the real scalar product does not couple the 0-component with the 1component of the Cauchy data, which are real orthogonal:

$$
(f, g)_{\mathbb{R}}=\langle f, P g\rangle=\left\langle f_{0}, P_{00} g_{0}\right\rangle+\left\langle f_{1}, P_{11} g_{1}\right\rangle .
$$

Remark 3.4. A time reversion map can be defined on $C_{c}^{\infty}\left(\mathbb{R}^{d+1}\right) / \sim$ using the map 15 on the right hand side of $\mathbf{9}$ but it depends on the choice of a Cauchy surface, whilst the time orientation is a global characteristic which enters in the definition of the symplectic structure and does not depend on any $\mathbf{\Sigma}$.

We can summarize in the following

Proposition 3.5. Let $M \subset \mathbb{R}^{d+1}$ be any globally hyperbolic subset of Minkowski space-time, $\boldsymbol{\Sigma}$ a Cauchy surface for $M$, then the local real Hilbert space $H(M)$, generated by functions with support in $M$, is isomorphic to the space of Cauchy data:

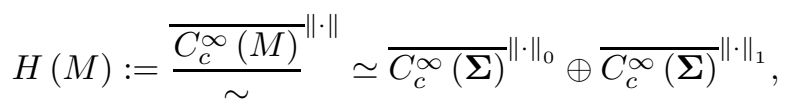

where the two norms are given by the real scalar products $\left(f_{0}, g_{0}\right)_{0}:=\left\langle f_{0}, P_{00} g_{0}\right\rangle,\left(f_{1}, g_{1}\right)_{1}:=$ $\left\langle f_{1}, P_{11} g_{1}\right\rangle$ and the direct sum is an orthogonal sum.

Remark 3.6. Whilst $H_{0}$ is a complex Hilbert space and its symplectic form is the imaginary part of the scalar product, the two isomorphic spaces in 18 are in general not invariant under $J$, therefore they are only real symplectic Hilbert spaces, with the corresponding symplectic forms given in Prop. 3.3

\section{Scalar Free Field}

\subsection{Causal Structure of the One-Particle Space}

The Hilbert space $H_{0}$ described in the previous section is the one-particle space of the scalar free field on $d+1$-dimensional Minkowski space-time. The quantum field and observable 
algebra are represented as operators on the Fock space constructed on it (Section 2); $H:=$ $H(M) \subset H_{0}$ is the local space associated to an open globally hyperbolic subset $M \subset \mathbb{R}^{d+1}$ of the $d+1$-dimensional Minkowski space-time.

As we have a natural association of CCR-subalgebras $\mathfrak{A}(V)$ to real subspaces $V \subset H$, the local structure of the algebra of observables comes from the corresponding one on the one-particle space $H$.

Definition 4.1. For every open subset $O \subset M$ we define the local subspace $H(O) \subset H$ as

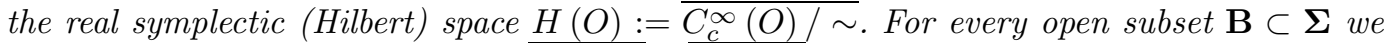
define the local subspace $\mathbf{H}(\mathbf{B}):=\overline{C_{c}^{\infty}(\mathbf{B})} \oplus \overline{C_{c}^{\infty}(\mathbf{B})}$.

The local subspaces are not complex spaces because they are not invariant under the multiplication by $i$. According to the isomorphisms of Prop. 3.3 and 3.5

$$
H(C(\mathbf{B}))=\overline{C_{c}^{\infty}(C(\mathbf{B})) / \sim}=\bigcap_{A \supset \mathbf{B}} H(A) \simeq \overline{C_{c}^{\infty}(\mathbf{B}) \otimes \mathbb{R}^{2}}=\mathbf{H}(\mathbf{B}) .
$$

The second equality follows from the fact that for any open $A$ such that $\mathbf{B} \subset A \subset C(\mathbf{B})$, obviously $H(A) \subset H(C(\mathbf{B}))$ but also $H(A) \supset H(C(\mathbf{B})) \simeq \mathbf{H}(\mathbf{B})$ with the same argument used in the proof of Prop. 3.3. Using the Cauchy data formulation one can exploit eq. 2 and thus

$$
H\left(C(\mathbf{B})^{c}\right) \simeq \mathbf{H}(\boldsymbol{\Sigma} \backslash \overline{\mathbf{B}}) .
$$

\subsection{Haag Duality and One-Particle-Space Duality}

Having defined a local structure of the one-particle space $H \subset H_{0}$, the CCR-algebra $\mathfrak{A}(H, \sigma)$ gains a local structure. If we then consider the Fock representation on the Hilbert space $\Gamma\left(H_{0}\right)$, we can define a local net of von Neumann algebras $O \mapsto \mathfrak{R}(O)$.

Definition 4.2. For every open subset $O \subset M$, the local von Neumann algebra $\mathfrak{R}(O)$ in Fock representation is the algebra $\mathfrak{R}(H(O))$ (eq. 1), i.e. the algebra generated by the Weyl operators $W(f)$ for all smooth $f$ with compact support in $O$.

From the properties of Fock representation and of the one-particle space, we easily deduce the properties of the net of local von Neumann algebras $\mathfrak{R}$. E.g., the algebra $\mathfrak{R}(C(\mathbf{B}))$ associated to a diamond $C(\mathbf{B})$ coincides with the algebra $\mathfrak{R}(\mathbf{B}):=\mathfrak{R}(\mathbf{H}(\mathbf{B})$ ) associated to its basis: $\mathfrak{R}(\mathbf{B})=\bigwedge_{A \supset \mathbf{B}} \mathfrak{R}(A)=\mathfrak{R}(C(\mathbf{B}))$, where $A$ ranges over the set of open subsets of $\mathbb{R}^{d+1}$.

Abstract Haag duality (Th. 2.1) implies that Haag duality, even in its relative form, is equivalent to a property of the local one-particle Hilbert space that we call one-particle space duality. It is again a maximality property with respect to locality, namely the fact that the local space $H\left(O^{c}\right)$ associated to the causal complement $O^{c}$ of a region $O$ of space-time coincides with the symplectic complement $H(O)^{c}$ of the local space associated to $O$.

Definition 4.3. The local subspaces $H(O)$ or $\mathbf{H}(\mathbf{B}) \subset H$ are said to satisfy one-particle space duality iff, respectively,

$$
H\left(O^{c}\right)^{c}=H(O), \quad \mathbf{H}(\boldsymbol{\Sigma} \backslash \overline{\mathbf{B}})^{c}=\mathbf{H}(\mathbf{B}) .
$$

Remark 4.4. The previous equations are not equivalent to

$$
H(O)^{c}=H\left(O^{c}\right), \quad \mathbf{H}(\mathbf{B})^{c}=\mathbf{H}(\mathbf{\Sigma} \backslash \overline{\mathbf{B}}) .
$$

as the symplectic complement is not in general an involutive map on closed subspaces: $V^{\text {cc }}$ is in general not equal to $V$ even for closed $V \subset H$. 
Such a property is expected to hold only for a certain class of regions; indeed, some regularity of the region $O$ is needed to prove the following property that will be used in the proof of Th. 4.10.

Definition 4.5. We say that a local subspace $\mathbf{H}(\mathbf{B})$ or $H(O)$ satisfies outer regularity if:

$$
\mathbf{H}(\mathbf{B})=\bigcap_{\mathbf{A} \supset \overline{\mathbf{B}}} \mathbf{H}(\mathbf{A}), \quad H(O)=\bigcap_{A \supset \bar{O}} H(A)
$$

where $\mathbf{A}$ ranges over the open neighborhoods of $\overline{\mathbf{B}}$ in $\mathbf{\Sigma}$ and $A$ over the open diamonds containing $\bar{O}$ in $M$.

A similar definition can be given for local algebras. We quote the main statement here and refer to App. $\mathrm{A}$ for proofs and references.

Proposition 4.6. Let $\mathbf{B} \subset \boldsymbol{\Sigma}$ be the basis of a double cone $O \subset \mathbb{R}^{d+1}$, then the local spaces $\mathbf{H}(\mathbf{B}) \simeq H(O)$ satisfy outer regularity.

A simple Lemma leads us to the main theorem:

Lemma 4.7. Relative duality $\mathfrak{R}\left(O^{c}\right)^{c}=\mathfrak{R}(O)$ is equivalent to one-particle space duality $H\left(O^{c}\right)^{c}=H(O)$ or, in terms of Cauchy data, $\mathbf{H}(\boldsymbol{\Sigma} \backslash \overline{\mathbf{B}})^{c}=\mathbf{H}(\mathbf{B})$.

Proof. From Prop. 2.4] $\mathfrak{R}\left(H\left(O^{c}\right)\right)^{c}=\mathfrak{R}\left(H\left(O^{c}\right)^{c}\right)$; to use Cauchy data, apply the isomorphisms in 19 and 20

We state now relative Haag duality in some relevant cases:

Theorem 4.8. Let the ambient space $M$ be any open globally hyperbolic submanifold of Minkowski space-time $\mathbb{R}^{d+1}$ with $d \geq 2, O$ any double cone with $\bar{O} \subset M$, then the algebra of observables for the free scalar field in the representation of the vacuum state satisfies relative duality:

$$
\mathfrak{R}\left(O^{c}\right)^{c}=\mathfrak{R}(O)
$$

The dual relation (cf. Rem. 4.4)

$$
\mathfrak{R}(O)^{c}=\mathfrak{R}\left(O^{c}\right)
$$

holds in the following cases

- the field is massive

- the field is massless, $M \equiv V_{+}$and $O=C(\mathbf{B})$ is any double cone with basis $\mathbf{B} \subset \mathbf{\Sigma}$, where $\boldsymbol{\Sigma}=\left\{x \in \mathbb{R}^{d+1}: x_{0} \geq 0, x^{2}=c \in \mathbb{R}_{+}\right\}$is an hyperboloid in $V_{+}$.

Proof. The proof follows from Lemma 4.7 and the theorems below on one-particle space duality: Th. 4.10 and, for the dual relation, considerations in Subsection 4.4.1 and Th. 4.11.

\subsection{Duality for a Double Cone $O$}

We want to prove that $H\left(O^{c}\right)^{c}=H(O)$ for any double cone $O \subset \bar{O} \subset M \subset \mathbb{R}^{d+1}$. We use a Cauchy surface: let $O_{1}$ be a double cone such that $\bar{O} \subset O_{1} \subset M$, choose a Cauchy surface $\boldsymbol{\Sigma}$ containing the basis $\mathbf{B}_{1}$ of $O_{1}=C\left(\mathbf{B}_{1}\right) \subset \mathbb{R}^{d+1}$ (and the basis $\mathbf{B}$ of $O$ ). Such a Cauchy surface can be explicitly constructed in the cases of interest $\left(M=V_{+}\right)$and its existence has been proved in full generality in [5]. In terms of Cauchy data the previous equality is 
equivalent to $\mathbf{H}\left(\boldsymbol{\Sigma} \backslash \overline{\mathbf{B}}^{c}\right)^{c}=\mathbf{H}(\mathbf{B})$. Following [22] 23], we prove duality via outer regularity, by proving that

$$
\mathbf{H}(\boldsymbol{\Sigma} \backslash \overline{\mathbf{B}})^{c}=\bigcap_{\mathbf{A} \supset \overline{\mathbf{B}}} \mathbf{H}(\mathbf{A}) .
$$

Using the symplectic form $\sigma$, we can define in a natural way an immersion

$$
\psi: H \supset C_{c}^{\infty}(\boldsymbol{\Sigma}) \otimes \mathbb{R}^{2} \hookrightarrow C^{-\infty}(\boldsymbol{\Sigma}) \otimes \mathbb{R}^{2}
$$

with $\langle\psi(f), g\rangle:=\sigma(f, g)$ for any $g \in C_{c}^{\infty}(\boldsymbol{\Sigma}) \otimes \mathbb{R}^{2}$. This map on $C_{c}^{\infty}(\boldsymbol{\Sigma}) \otimes \mathbb{R}^{2}$ is continuous, because $f_{n} \stackrel{\|\cdot\|}{\rightarrow} f$ implies $\sigma\left(f_{n}, g\right) \rightarrow \sigma(f, g)$ for any $g \in C_{c}^{\infty}(\boldsymbol{\Sigma}) \otimes \mathbb{R}^{2}$, i.e. $\psi\left(f_{n}\right) \rightarrow \psi(f)$ in the topology of distributions (weak convergence); it can therefore be extended to the whole Hilbert space $H$. It is injective, because if $\sigma(f, g)=0$ for any $g \in C_{c}^{\infty}(\boldsymbol{\Sigma}) \otimes \mathbb{R}^{2}$, which is dense in $H$, then $f=0$. Restricted to $C_{c}^{\infty}(\boldsymbol{\Sigma}) \otimes \mathbb{R}^{2}$, the map $\psi$ is just the composition of the map $f_{0} \oplus f_{1} \stackrel{U}{\mapsto}-f_{1} \oplus f_{0}$ with the canonical injection $C_{c}^{\infty}(\boldsymbol{\Sigma}) \otimes \mathbb{R}^{2} \hookrightarrow C^{-\infty}(\boldsymbol{\Sigma}) \otimes \mathbb{R}^{2}$ : whenever $f, g \in C_{c}^{\infty}(\boldsymbol{\Sigma}) \otimes \mathbb{R}^{2}$, according to 10.

$$
\langle U f, g\rangle=\left\langle-f_{1} \oplus f_{0}, g_{0} \oplus g_{1}\right\rangle=\left\langle f_{0}, g_{1}\right\rangle-\left\langle f_{1}, g_{0}\right\rangle=\sigma(f, g)=\langle\psi(f), g\rangle .
$$

Remark 4.9. The former construction shows that a general element $f=f_{0} \oplus f_{1} \in H$, as a limit of functions, is a distribution $f_{0} \oplus f_{1} \in \mathcal{S}^{\prime} \oplus \mathcal{S}^{\prime}$ and $\psi(f)=-f_{1} \oplus f_{0}$.

Theorem 4.10. For any double cone $O$ such that $\bar{O} \subset M$ with basis $\mathbf{B}$, the local subspaces $H(O) \simeq \mathbf{H}(\mathbf{B})$ satisfy (relative) duality:

$$
H\left(O^{c}\right)^{c}=H(O), \quad \mathbf{H}(\boldsymbol{\Sigma} \backslash \overline{\mathbf{B}})^{c}=\mathbf{H}(\mathbf{B}) .
$$

Proof. Let $f \in \mathbf{H}(\boldsymbol{\Sigma} \backslash \overline{\mathbf{B}})^{c}$, then, by definition of the relative symplectic complement of $\mathbf{H}(\boldsymbol{\Sigma} \backslash \overline{\mathbf{B}})$, the distribution $\psi(f)$ has support in $\overline{\mathbf{B}} \subset \mathbf{B}_{1}$. Now, if we restrict our attention to the region $O_{1}$, the Cauchy surface $\boldsymbol{\Sigma}$ can be identified with $\mathbb{R}^{d}$ and we can use the known results on Minkowski space-time and time-0 Cauchy surface.

To find regular functions approximating $f \in \mathbf{H}(\boldsymbol{\Sigma} \backslash \overline{\mathbf{B}})^{c}$ we use convolutions with regular functions $\rho_{n}$ approximating Dirac's $\delta$. Let $\rho \in C_{c}^{\infty}\left(\mathbb{R}^{d}\right)$ be an even function such that $\int \rho(\mathbf{x}) d \mathbf{x}=1$, and $C_{c}^{\infty}\left(\mathbb{R}^{d}\right) \ni \rho_{n}(x):=n^{d} \rho(n x)$, then define $f_{n}:=\rho_{n} * \psi(f)$. The functions $f_{n}$ are in $C_{c}^{\infty}\left(\mathbb{R}^{d}\right) \otimes \mathbb{R}^{2}$ and have for large $n$ support in $\mathbf{B}_{1}$. Therefore, for $n$ big enough, as elements of $C_{c}^{\infty}(\boldsymbol{\Sigma}) \otimes \mathbb{R}^{2}$, they belong to $\mathbf{H}\left(\mathbf{B}_{\mathbf{1}}\right) \simeq H\left(O_{1}\right)$. It can be proved, using Fourier transform (see [22]) that $f_{n} \rightarrow f$ in $\mathbf{H}\left(\mathbf{B}_{\mathbf{1}}\right)$. Moreover, for any open set $\mathbf{A} \ni 0$, $f_{n} \in \mathbf{H}(\mathbf{B}+\mathbf{A})$ for large $n$, because $\operatorname{supp} f_{n}=\operatorname{supp} \psi(f)+\operatorname{supp} \rho_{n} \subset \mathbf{B}+\mathbf{A}$. Therefore, $f=\lim f_{n}$ is in $\bigcap_{\mathbf{A}} \mathbf{H}(\mathbf{B}+\mathbf{A})$, where $\mathbf{A}$ ranges in the set of open neighborhoods of 0 in $\mathbb{R}^{d}$. Finally, by outer regularity, Prop. 4.6] we conclude that $f \in \mathbf{H}(\mathbf{B})$.

\subsection{Duality for $O^{c}$}

We address now the other duality relation $\mathfrak{R}(O)^{c}=\mathfrak{R}\left(O^{c}\right)$, where the role of $O$ and $O^{c}$ are interchanged, recalling Rem. 4.4 This relation is equivalent to $H(O)^{c}=H\left(O^{c}\right)$ (or $\left.H(O)^{\prime} \cap H(M)=H\left(O^{\prime} \cap M\right)\right)$. Difficulties arise because the elements of $H(O)^{c}$ have support in $O^{c}$, which is not compact if $M$ is not, and possibly because of the topology of $O^{c}$. We do not prove this relation in generality but we specialize now to the case $M=V_{+}$ and distinguish the massive and massless cases.

\subsubsection{The massive case: $H\left(V_{+}\right)=H_{0}$}

In the massive case, the algebra associated to the forward (or backward) light-cone is already the total algebra of observables or, in terms of one-particle spaces, $H\left(V_{+}\right)=H\left(\mathbb{R}^{d+1}\right)=H_{0}$ is the whole Hilbert space; see Theorems 1 and 2 in [26]. 
In such case, the relative symplectic complement coincides with the symplectic complement $\left(V^{c}=V^{\prime} \forall V \in \operatorname{subsp}\left(H\left(V_{+}\right)\right)\right)$and is therefore involutive, $V^{c c}=V$. Duality for $O^{c}$ follows trivially from duality for $O$ because, taking the dual of both sides in eq. 24] where $\mathbf{H}(\boldsymbol{\Sigma} \backslash \overline{\mathbf{B}})^{c} \equiv \mathbf{H}(\boldsymbol{\Sigma} \backslash \overline{\mathbf{B}})^{\prime}$, we obtain $\mathbf{H}(\boldsymbol{\Sigma} \backslash \overline{\mathbf{B}})=\mathbf{H}(\mathbf{B})^{\prime}$.

\subsubsection{The massless case}

In the massless case with odd space dimension $d, H\left(V_{+}\right)$has a big symplectic complement $H\left(V_{+}\right)^{\prime}=H\left(V_{-}\right)$in $H_{0}$. This is a consequence of Huygens' principle and corresponds to time-like duality for the algebra of the light-cone: $\mathfrak{R}\left(V_{+}\right)^{\prime}=\mathfrak{R}\left(V_{-}\right)$(see [7]).

However, in the massless case, for any space-time dimension $d+1$, we can use conformal covariance and the consequent unitary equivalence of the algebras $\mathfrak{R}\left(V_{+}\right)$and $\mathfrak{R}\left(O_{1}\right)$, where $O_{1}$ is a double cone [20, thus reducing our ambient space to a relatively compact one.

A relativistic ray inversion map $\varphi_{0}: x \mapsto-x / x^{2}$ transforms a double cone $O_{1}$, which has the upper vertex in 0 and the lower vertex in $(-T, \mathbf{0})$, to the translated forward light cone $V_{+}+\left(\frac{1}{T}, \mathbf{0}\right)$ and its basis $\mathbf{B}_{1}=\left\{\left(-\frac{T}{2}, \mathbf{x}\right):\|\mathbf{x}\|<\frac{T}{2}\right\}$ to the hyperboloid $\left\{x: x_{0} \geq 0, x^{2}=1 / T^{2}\right\}+\left(\frac{1}{T}, \mathbf{0}\right)$. Of course, as $\varphi_{0}^{-1}=\varphi_{0}$, also the converse is true and composing the inversion map $\varphi_{0}$ with suitable translations a conformal map $\varphi$ can be found such that $O_{1}:=\varphi\left(V_{+}\right)$is a double cone and, for any double cone $O$ with closure in $V_{+}$, $\varphi(O)$ is a double cone with closure in $O_{1}$. For further simplicity, we assume that the vertices of $O$ and the vertex of $V_{+}$lie on the same straight line. In this case an hyperboloid $\boldsymbol{\Sigma}=\left\{x: x_{0} \geq 0, x^{2}=c \in \mathbb{R}_{+}\right\}$can be found such that $O=C(\mathbf{B})$ with $\mathbf{B} \subset \boldsymbol{\Sigma}$ and the map $\varphi$ can be chosen so that the basis of $O_{1}, \mathbf{B}_{1}=\varphi(\boldsymbol{\Sigma})$, is lying on the time-0 surface $\mathbb{R}^{d} \subset \mathbb{R}^{d+1}$. $\mathbf{B}_{1} \subset \mathbb{R}^{d}$ is obviously a Cauchy surface for $O_{1}$, whilst $\mathbb{R}^{d}$ is the standard time- 0 Cauchy surface for $\mathbb{R}^{d+1}$.

Theorem 4.11. Let $\boldsymbol{\Sigma}=\left\{x \in \mathbb{R}^{d+1}: x_{0} \geq 0, x^{2}=c \in \mathbb{R}_{+}\right\}$be an hyperboloid in $V_{+}, O=$ $C(\mathbf{B})$ any double cone with basis $\mathbf{B} \subset \boldsymbol{\Sigma}$, then the local subspaces $H\left(O^{c}\right) \simeq \mathbf{H}(\boldsymbol{\Sigma} \backslash \overline{\mathbf{B}})$ satisfy (relative) duality:

$$
H\left(O^{c}\right)=H(O)^{c}, \quad \mathbf{H}(\boldsymbol{\Sigma} \backslash \overline{\mathbf{B}})=\mathbf{H}(\mathbf{B})^{c} .
$$

Proof. As explained above, using a conformal transformation, we can reduce the problem to the following: let $O=C(\mathbf{B})$ and $O_{1}=C\left(\mathbf{B}_{1}\right)$ be two double cones with basis $\mathbf{B}$ and $\mathbf{B}_{1}$ such that $\overline{\mathbf{B}} \subset \mathbf{B}_{1} \subset \mathbf{\Sigma} \equiv \mathbb{R}^{d}$, then we want to prove that $H(\mathbf{B})^{\prime} \cap H\left(\mathbf{B}_{1}\right)=H\left(\mathbf{B}^{\prime} \cap \mathbf{B}_{1}\right)$. Let $f \in \mathbf{H}(\mathbf{B})^{\prime} \cap \mathbf{H}\left(\mathbf{B}_{\mathbf{1}}\right)$, applying the map 22 we have a distribution $\psi(f) \in C^{-\infty}\left(\mathbb{R}^{d}\right) \otimes \mathbb{R}^{2}$ whose support must be in $\overline{\mathbf{B}_{1}}$ because $f \in \mathbf{H}\left(\mathbf{B}_{\mathbf{1}}\right)$ and in $\mathbf{B}^{c}$ because $f \in \mathbf{H}(\mathbf{B})^{\prime}$, i.e. $\operatorname{supp} f \subset \overline{\mathbf{B}_{1}} \backslash \mathbf{B}$. Let $\rho, \rho_{n} \in C_{c}^{\infty}\left(\mathbb{R}^{d}\right)$ be as in Sec. 4.3 $f_{n}:=\rho_{n} * \psi(f)$. The functions $f_{n}$ are in $C_{c}^{\infty}\left(\mathbb{R}^{d}\right) \otimes \mathbb{R}^{2}$ and, for any open neighborhood $\mathbf{A} \subset \mathbb{R}^{d}$ of 0 , have support in $\left(\mathbf{B}_{1} \backslash \overline{\mathbf{B}}\right)+\mathbf{A}$, therefore belong to $\mathbf{H}\left(\left(\mathbf{B}_{1} \backslash \overline{\mathbf{B}}\right)+\mathbf{A}\right)$. As in Sec. 4.3 $f_{n} \rightarrow f$ in $\mathbf{H}\left(\mathbb{R}^{\mathbf{d}}\right)$, hence $f=\lim f_{n} \in \bigcap_{\mathbf{A}} \mathbf{H}\left(\left(\mathbf{B}_{\mathbf{1}} \backslash \overline{\mathbf{B}}\right)+\mathbf{A}\right)$, and one-particle space duality follows from outer regularity for the non contractible region $B_{1} \backslash \bar{B}$, Prop. A.2 in App. A

\section{$5 \quad$ Free Electromagnetic Field}

Let the ambient space $M \subset \mathbb{R}^{d+1}$ be an open globally hyperbolic maximal submanifold, as in Sect. 3. with the further requirement that it is contractible to avoid cohomological problems. We denote by $\Omega^{*}(M)$ the set of differential forms, with a prefix $Z^{d}, Z^{\delta}$ for the closed forms with respect to $d$ or its formal adjoint $\delta$ and similarly $B^{d}, B^{\delta}$ for the exact forms (cf. App. B for more details). 


\subsection{Quantum Free Electromagnetic Field}

The quantum electromagnetic field $F$ is an operator valued continuous functional on the test space of 2-forms satisfying Maxwell equations, i.e. $d F=0$ and $\delta F=0$. From the first equation, as the 2-cohomology of $M$ is trivial (the cohomology of currents coincides with the cohomology of forms, generalized De Rham's Theorem, Chap. IX in [27]), it is the external derivative of the (equivalence class $[A]$ of the) electromagnetic potential $A$ : $F=d A$. Two potentials $A, A^{\prime}$ are equivalent iff $d A=d A^{\prime}$; it follows that $A$ has to be considered as an operator valued continuous functional on the test space of $\delta$-closed (or $\delta$-boundary) 1-forms, because triviality of the cohomology implies that $\frac{\Omega^{\prime 1}(M)}{Z^{d} \Omega^{\prime 1}(M)}=\left(Z^{\delta} \Omega_{c}^{1}(M)\right)^{\prime}$. Let $a \in Z^{\delta} \Omega_{c}^{1}(M)$, then there exists $f \in \Omega_{c}^{2}(M)$ such that $a=\delta f$ and $A(a)=A(\delta f)=$ $d A(f)=F(f)$ is the electromagnetic field smeared with the test function $f$.

The antisymmetric bilinear form on $Z^{\delta} \Omega_{c}^{1}(M)$ or $\Omega_{c}^{2}(M)$ given by (cf. [22])

$$
\sigma\left(a_{1}, a_{2}\right):=\left\langle a_{1}, E a_{2}\right\rangle=-\int a_{1} \wedge * E a_{2}=-\int \delta f_{1} \wedge * E \delta f_{2}
$$

is degenerate. We take the quotient of the space of 1 -forms with respect to the degeneracy space, i.e. with respect to the equivalence relation: $a \sim 0$ iff $\sigma(a, \cdot)=0$, or, equivalently, $d E a=0$. We indicate again with $\sigma$ the induced symplectic form on $Z^{\delta} \Omega_{c}^{1}(M) / \sim$, which becomes in this way a symplectic space. In terms of the 2 -form test function $f$, the symplectic space is $\Omega_{c}^{2}(M) / \sim$, where $f \sim 0$ iff $d E f=0$ and $\delta E f=0$.

As in the scalar case, we can define in a natural way a complex structure on $H_{0}:=$ $H\left(\mathbb{R}^{d+1}\right)$ with a square root $J$ of $\mathbf{- 1}$ given by multiplication in Fourier transform by $i \epsilon\left(p_{0}\right)$ : $\widehat{J a}(p):=i \epsilon\left(p_{0}\right) \widehat{a}(p)$. As $J$ is not a local operator, $H(M)$ is in general not invariant under $J$. The propagator $E: \Omega_{c}^{k}\left(\mathbb{R}^{d+1}\right) \rightarrow \Omega^{k}\left(\mathbb{R}^{d+1}\right)$, when restricted to $\Omega_{c}^{k}(M)$, gives the propagator on $M$. The Fock representation corresponds to the positive semi-definite inner product on $Z^{\delta} \Omega_{c}^{1}\left(\mathbb{R}^{d+1}\right)$ :

$$
\left(a_{1}, a_{2}\right)_{\mathbb{R}}:=\left\langle a_{1}, P a_{2}\right\rangle=-\int a_{1} \wedge * P a_{2}=-\int \delta f_{1} \wedge * P \delta f_{2},
$$

where $*$ is the Hodge- $*$ operator and $P=J E$. The 0 -norm elements are those equivalent to 0 , therefore the inner product is positive definite on the quotient space and the completion is the one-particle complex Hilbert space

$$
H_{0}:=H\left(\mathbb{R}^{d+1}\right)=\frac{\overline{Z^{\delta} \Omega_{c}^{1}\left(\mathbb{R}^{d+1}\right)}}{\sim} \simeq \frac{\overline{\Omega_{c}^{2}\left(\mathbb{R}^{d+1}\right)}}{\sim} .
$$

The symplectic form $\sigma(a, b)=\langle a, E b\rangle$ can be extended to the closure and the vacuum state is the Fock state $\omega$ on the CCR-algebra $\mathfrak{A}\left(H_{0}, \sigma\right)$ whose two point function is the complex scalar product

$$
\omega(a, b)=(a, b)_{\mathbb{R}}+i \sigma(a, b) .
$$

\subsection{Cauchy Data}

Let $\boldsymbol{\Sigma} \subset M \subset \mathbb{R}^{d+1}$ be a Cauchy surface for $M$ and $\rho_{i}$ the corresponding operators (cf. 401). As $M$ is homeomorphic to $\boldsymbol{\Sigma} \times \mathbb{R}$, our assumption that $M$ is contractible is equivalent to contractibility of $\boldsymbol{\Sigma}$ (and of every Cauchy surface). The electromagnetic field $F$ and the potential $[A]$, which satisfy the wave equation, are determined by their Cauchy data on $\boldsymbol{\Sigma}$ and it is well known that the values of the field and its normal derivative on $\boldsymbol{\Sigma}$ are not independent. We now choose to work with the potential $[A] \in \frac{\Omega^{\prime 1}(M)}{Z^{d} \Omega^{\prime 1}(M)}$ and the test 1forms $[a]$. Given $[a] \in Z^{\delta} \Omega_{c}^{1}(M) / \sim$ then $[E a] \in Z^{\delta} \Omega^{1}(M) / \sim$ and $\delta d E a=0$, because on $Z^{\delta} \Omega^{1}(M)$ the equations $\square a \equiv(d \delta+\delta d) a=0$ and $\delta d a=0$ are equivalent and $\square E=0$. The Cauchy data for $E a$ can be chosen to be $[\mathbf{a}]:=\left[\rho_{0} E a\right] \in \Omega_{c}^{1}(\boldsymbol{\Sigma}) / Z^{d} \Omega_{c}^{1}(\boldsymbol{\Sigma})$, where $\mathbf{a} \sim 0$ iff 
$d \mathbf{a}=0$, and $\mathbf{e}:=\rho_{1} E a \in Z^{\delta} \Omega_{c}^{1}(\boldsymbol{\Sigma})$, as shown in [11. We quote here the existence statement, [11] Prop. 2:

Proposition 5.1. For any $\mathbf{a}, \mathbf{e} \in \Omega_{c}^{1}(\boldsymbol{\Sigma})$ with $\delta \mathbf{e}=0$, there exists $a \in Z^{\delta} \Omega^{1}(M)$ such that $\square a=0$ (i.e. $\delta d a=0$ ), $\rho_{0} a=\mathbf{a}$ and $\rho_{1} a=\mathbf{e}$.

The following is a uniqueness statement, 11] Prop. 3.

Proposition 5.2. For $i=1,2$, let $a_{i} \in \Omega^{1}(M)$ satisfy $\delta d a_{i}=0$ and $\rho_{0} a_{i}=\mathbf{a}_{i}, \rho_{1} a_{i}=\mathbf{e}_{i}$ (so that $\delta \mathbf{e}_{i}=0$ ), then $a_{1} \sim a_{2}$ iff $\mathbf{e}_{1}=\mathbf{e}_{2}$ and $\mathbf{a}_{1} \sim \mathbf{a}_{2}$.

Notice that the proofs hold in the present context, where our globally hyperbolic manifold is a subset of Minkowski space-time and we do not require the Cauchy surface to be compact.

Moreover, like in the scalar case (cf. eq. 17), considering the effect of change of time orientation one can conclude that the real scalar product does not couple the 0 -component with the 1-component of the Cauchy data:

$$
\left(a_{1}, a_{2}\right)_{\mathbb{R}}=\left\langle a_{1}, P a_{2}\right\rangle=\left\langle\mathbf{a}_{1}, P_{00} \mathbf{a}_{2}\right\rangle+\left\langle\mathbf{e}_{1}, P_{11} \mathbf{e}_{2}\right\rangle .
$$

We can then state the following

Theorem 5.3. There is an isomorphism of real Hilbert spaces between the space of (equivalence classes of) covariant potentials and the space of (equivalence classes of) Cauchy data:

$$
\begin{array}{ccccc}
\overline{\frac{Z^{\delta} \Omega_{c}^{1}(M)}{c}} & \simeq & \overline{\frac{\Omega_{c}^{1}(\boldsymbol{\Sigma})}{Z^{d} \Omega_{c}^{1}(\boldsymbol{\Sigma})}} & \oplus & \overline{Z^{\delta} \Omega_{c}^{1}(\boldsymbol{\Sigma})} \\
{[a]} & \leftrightarrow & {[\mathbf{a}]} & \oplus & \mathbf{e}
\end{array}
$$

where $\mathbf{a}=\rho_{0} a$ and $\mathbf{e}=\rho_{1} a$ for $a \in \frac{Z^{\delta} \Omega_{c}^{1}(M)}{\sim}$ and the closures are with respect to the scalar product [29. The symplectic form in terms of Cauchy data is

$$
\sigma\left(a_{1}, a_{2}\right)=\int_{\Sigma} j^{*}\left(E a_{1} \wedge * d E a_{2}-E a_{2} \wedge * d E a_{1}\right)=\left\langle\mathbf{a}_{1}, \mathbf{e}_{2}\right\rangle-\left\langle\mathbf{e}_{1}, \mathbf{a}_{2}\right\rangle
$$

Proof. The isomorphism is an immediate consequence of Prop. 5.1 and 5.2 and of the form of the scalar product 29

The expression of the symplectic form 31 follows by eq. 42 in App. B noting that the second term in the sum vanishes when the forms belong to $Z^{\delta} \Omega_{c}^{1}(M)$, because then $\rho_{1} *=* j^{*} \delta$ is zero. Alternatively, using Stokes' theorem, one can also verify that the integral in 31 does not depend on $\boldsymbol{\Sigma}$ and reduce the proof of 31 to the known expression in the case in which $\boldsymbol{\Sigma}$ is the time-0 surface. The difference of the integrals in 31 calculated on two (non intersecting) Cauchy surfaces can be computed as the integral $\int d\left(E a_{1} \wedge * d E a_{2}-E a_{2} \wedge * d E a_{1}\right)$ on the region enclosed by the two surfaces; this integral is 0 because $\delta d E a_{i}=0$.

\subsection{Local Algebras}

As in the scalar case, the local algebras are associated to the local spaces defined in the natural way: $H(O)=\overline{\left[Z^{\delta} \Omega_{c}^{1}(O)\right]}$. Using the Cauchy data, eq. 31] becomes for local subspaces

$$
H(C(\mathbf{B})) \simeq \mathbf{H}(\mathbf{B})=\overline{\left[\Omega_{c}^{1}(\mathbf{B})\right] \oplus Z^{\delta} \Omega_{c}^{1}(\mathbf{B})} .
$$

The CCR-algebra $\mathfrak{A}\left(H_{0}, \sigma\right)$ associated to the symplectic space $H_{0}$ (eq. 27) is the algebra of observables for the electromagnetic free field and it has the local structure given by the local structure of $H_{0}$ in $32 \mathfrak{A}(O):=\mathfrak{A}(H(O))$.

The vacuum state is the state defined by the 2-point function [28] via the real scalar product [26] as in the scalar case. In the corresponding GNS-representation on the Fock 
space $\Gamma\left(H_{0}\right)$ the weak closure of the local algebras gives a net of von Neumann algebras $O \mapsto \mathfrak{R}(O)$.

Our main theorem is then:

Theorem 5.4. Let $M$ be an open globally hyperbolic contractible submanifold of Minkowski space-time $\mathbb{R}^{d+1}$ with $d \geq 2$; for any double cone $O$ with $\bar{O} \subset M$, the algebra of observables for the free electromagnetic field in the representation of the vacuum state satisfies relative duality:

$$
\mathfrak{R}\left(O^{c}\right)^{c}=\mathfrak{R}(O)
$$

The proof will follow from Th. 5.9 below and Lemma 4.7 which holds also in this case.

\subsection{One-Particle Space Duality for a Double Cone $O$}

As for $M$, triviality of the cohomology of $\boldsymbol{\Sigma}$ implies that

$$
\frac{\Omega^{\prime 1}(\boldsymbol{\Sigma})}{Z^{d} \Omega^{\prime 1}(\boldsymbol{\Sigma})}=\left(Z^{\delta} \Omega_{c}^{1}(\boldsymbol{\Sigma})\right)^{\prime}
$$

Let us define via the symplectic form a continuous immersion of the Hilbert space $H$ into a space of (equivalence classes of) 1-currents (distribution valued 1-forms).

$$
\psi: H \supset \frac{\Omega_{c}^{1}(\boldsymbol{\Sigma})}{Z^{d} \Omega_{c}^{1}(\boldsymbol{\Sigma})} \oplus Z^{\delta} \Omega_{c}^{1}(\boldsymbol{\Sigma}) \hookrightarrow \frac{\Omega^{\prime 1}(\boldsymbol{\Sigma})}{Z^{d} \Omega^{\prime 1}(\boldsymbol{\Sigma})} \oplus Z^{\delta} \Omega^{\prime 1}(\boldsymbol{\Sigma}) .
$$

Using eq. 31 one first defines $\psi=\psi_{0} \oplus \psi_{1}$ on $\frac{\Omega_{c}^{1}(\boldsymbol{\Sigma})}{Z^{d} \Omega_{c}^{1}(\boldsymbol{\Sigma})} \oplus Z^{\delta} \Omega_{c}^{1}(\boldsymbol{\Sigma})$ as the identification of 1-forms $a_{1}=\left[\mathbf{a}_{1}\right] \oplus \mathbf{e}_{1}$ with distributions acting on $a_{2}=\left[\mathbf{a}_{2}\right] \oplus \mathbf{e}_{2} \in \frac{\Omega_{c}^{1}(\boldsymbol{\Sigma})}{Z^{d} \Omega_{c}^{1}(\boldsymbol{\Sigma})} \oplus Z^{\delta} \Omega_{c}^{1}(\boldsymbol{\Sigma})$ via the canonical scalar product: it is the direct sum of the maps $\psi_{0}: \frac{\Omega_{c}^{1}(\boldsymbol{\Sigma})}{Z^{d} \Omega_{c}^{1}(\boldsymbol{\Sigma})} \hookrightarrow \frac{\Omega^{\prime 1}(\boldsymbol{\Sigma})}{Z^{d} \Omega^{\prime 1}(\boldsymbol{\Sigma})}=$ $\left(Z^{\delta} \Omega_{c}^{1}(\boldsymbol{\Sigma})\right)^{\prime}$ and $\psi_{1}: Z^{\delta} \Omega_{c}^{1}(\boldsymbol{\Sigma}) \hookrightarrow Z^{\delta} \Omega^{\prime 1}(\boldsymbol{\Sigma})=\left(\frac{\Omega_{c}^{1}(\boldsymbol{\Sigma})}{Z^{d} \Omega_{c}^{1}(\boldsymbol{\Sigma})}\right)^{\prime}$, with $\left\langle\psi_{0}([a]), \mathbf{e}\right\rangle=\langle\mathbf{a}, \mathbf{e}\rangle$, $\left\langle\psi_{1}(\mathbf{e}), \mathbf{a}\right\rangle=-\langle\mathbf{e}, \mathbf{a}\rangle$, so that $\left\langle\psi\left(a_{1}\right), a_{2}\right\rangle=\left\langle\mathbf{a}_{1}, \mathbf{e}_{2}\right\rangle-\left\langle\mathbf{e}_{1}, \mathbf{a}_{2}\right\rangle$. Then, by the continuity of $\sigma$ with respect to the norm of $H$, we know that $\psi$ is continuous from $H$ to the space of distributions and can be extended to the whole Hilbert space. It is injective because $\sigma$ is non-degenerate and $\frac{\Omega_{c}^{1}(\boldsymbol{\Sigma})}{Z^{d} \Omega_{c}^{1}(\boldsymbol{\Sigma})} \oplus Z^{\delta} \Omega_{c}^{1}(\boldsymbol{\Sigma})$ is dense in $H$.

As in Subsection 4.3 let $O_{1} \supset \bar{O}$ be a double cone such that $\overline{O_{1}} \subset M$, choose a Cauchy surface $\boldsymbol{\Sigma}$ containing the basis $\mathbf{B}_{1}$ of $O_{1}=C\left(\mathbf{B}_{1}\right) \subset \mathbb{R}^{d+1}$ (and the basis $\mathbf{B}$ of $O=C(\mathbf{B}))$. We prove that $\mathbf{H}(\boldsymbol{\Sigma} \backslash \overline{\mathbf{B}})^{c}=\mathbf{H}(\mathbf{B})$ by proving eq. 21 and using outer regularity. Let $a \in \mathbf{H}(\boldsymbol{\Sigma} \backslash \overline{\mathbf{B}})^{c}$, then, by definition of the relative symplectic complement of $\mathbf{H}(\boldsymbol{\Sigma} \backslash \overline{\mathbf{B}})$, the distribution $\psi(a)$ has support in $\overline{\mathbf{B}} \subset \mathbf{B}_{1}$, where the support of an equivalence class of distributions is defined in the following obvious way:

Definition 5.5. The support of an equivalence class of distributions $[\mathbf{a}] \in \frac{\Omega^{\prime 1}(\boldsymbol{\Sigma})}{Z^{d} \Omega^{\prime 1}(\boldsymbol{\Sigma})}$ is the complement of the union of all open sets $\mathbf{A} \subset \boldsymbol{\Sigma}$ such that $Z^{\delta} \Omega_{c}^{1}(\mathbf{A}) \subset \operatorname{ker}[\mathbf{a}]$ (cf. eq. 33).

Proposition 5.6. For every $[\mathbf{a}] \in \frac{\Omega^{\prime 1}(\mathbf{\Sigma})}{Z^{d} \Omega^{\prime 1}(\boldsymbol{\Sigma})}$, da is a well defined 2-current and

$$
\operatorname{supp}(d \mathbf{a})=\operatorname{supp}[\mathbf{a}]=\bigcap_{\mathbf{a} \in[\mathbf{a}]} \operatorname{supp}(\mathbf{a}) .
$$

Proof. The support of the distribution a is the complement of the union of the open sets $\mathbf{A}$ such that $\left.\mathbf{a}\right|_{\mathbf{A}}=0$, where the last equality means that $\langle\mathbf{a}, \mathbf{e}\rangle=0$ for every (not necessarily $\delta$-closed) form e with support in $\mathbf{A}$; similarly for $d \mathbf{a}$. The definition is not altered if the family of open sets $\mathbf{A}$ is restricted to the open balls. 
1. Let $\mathbf{A}$ be an open set, such that $\langle[\mathbf{a}], \mathbf{e}\rangle=0$ for every $\mathbf{e} \in Z^{\delta} \Omega_{c}^{1}(\mathbf{A})$. For every $\mathbf{f} \in$ $\Omega_{c}^{2}(\mathbf{A})$ we have $\langle[\mathbf{a}], \delta \mathbf{f}\rangle=\langle d \mathbf{a}, \mathbf{f}\rangle=0$, thus $\left.d \mathbf{a}\right|_{\mathbf{A}}=0$. It follows that $\operatorname{supp}(d \mathbf{a}) \subset \operatorname{supp}[\mathbf{a}]$.

2. Let $\mathbf{A}$ be an open set, if there is an $\mathbf{a} \in[\mathbf{a}]$ such that $\left.\mathbf{a}\right|_{\mathbf{A}}=0$, then $\left.[\mathbf{a}]\right|_{\mathbf{A}}=0$. This implies that $\operatorname{supp}[\mathbf{a}] \subset \bigcap_{\mathbf{a} \in[\mathbf{a}]} \operatorname{supp}(\mathbf{a})$.

3. Let $\mathbf{A}$ be an open ball in $\mathbb{R}^{d}$, such that $\left.d \mathbf{a}\right|_{\mathbf{A}}=0$. As $\mathbf{A}$ is contractible, there is a distribution $\phi: \mathbf{A} \rightarrow \mathbb{R}$ such that $\left.\mathbf{a}\right|_{\mathbf{A}}=d \phi$. A distribution cannot in general be extended to $\mathbb{R}^{d}$. Nevertheless, for any open ball $\mathbf{A}_{\mathbf{1}}$ such that $\overline{\mathbf{A}_{\mathbf{1}}} \subset \mathbf{A}$, there exists a continuous function $\varphi \in C^{0}\left(\overline{\mathbf{A}_{\mathbf{1}}}\right)$ such that $\left.\phi\right|_{\mathbf{A}_{\mathbf{1}}}$ is the $n$-th derivative of $\varphi,\left.\phi\right|_{\mathbf{A}_{\mathbf{1}}}=\left.\partial^{n} \varphi\right|_{\mathbf{A}_{\mathbf{1}}} \cdot \varphi$ can be extended to a (not necessarily continuous) function on $\mathbb{R}^{d} \cdot \phi^{\prime}=\partial^{n} \varphi$ is a distribution on $\mathbb{R}^{d}$ that coincides with $\phi$ on $\mathbf{A}_{\mathbf{1}}$, thus $\mathbf{a}_{1}:=\mathbf{a}-d \phi^{\prime}$ is such that $\left[\mathbf{a}_{1}\right]=[\mathbf{a}]$ and $\left.\mathbf{a}_{1}\right|_{\mathbf{A}_{\mathbf{1}}}=0$. Therefore $\mathbf{A}_{\mathbf{1}} \subset \bigcup_{\mathbf{a} \in[\mathbf{a}]}(\operatorname{supp}(\mathbf{a}))^{c}$ whenever $\overline{\mathbf{A}_{\mathbf{1}}} \subset \mathbf{A}$. This implies that $\bigcap_{\mathbf{a} \in[\mathbf{a}]} \operatorname{supp}(\mathbf{a}) \subset \operatorname{supp}(d \mathbf{a})$.

Remark 5.7. It is a cohomological problem to know if $\operatorname{supp}[\mathbf{a}]=\mathbf{K} \subset \mathbf{B}$, with $\mathbf{B}$ open, implies that there is a representative $\mathbf{a} \in \Omega^{\prime 1}(\mathbf{B})$. We have that supp $[\mathbf{a}] \subset \mathbf{B}$ implies $\operatorname{supp}(d \mathbf{a}) \subset \mathbf{B}$ and, if $d \mathbf{a}$ is trivial in the second cohomology group $H^{2}(\mathbf{B})$ of $\mathbf{B}$, then $d \mathbf{a} \in B^{d} \Omega^{\prime 2}(\mathbf{B})$; this means that there exists $\tilde{\mathbf{a}} \in \Omega^{\prime 1}(\mathbf{B})$ such that $d \mathbf{a}=d \tilde{\mathbf{a}}$, which implies that $[\tilde{\mathbf{a}}]=[\mathbf{a}]$.

When we restrict our attention to the double cone $O_{1}$, the Cauchy surface $\boldsymbol{\Sigma}$ can be identified with $\mathbb{R}^{d}$ and we can use the known results on Minkowski space-time and time-0 Cauchy surface, including outer regularity [22]. To find regular forms approximating $a \in \mathbf{H}(\boldsymbol{\Sigma} \backslash \overline{\mathbf{B}})^{c}$ we use convolutions with regular functions $\rho_{n}$ approximating $\delta$.

Proposition 5.8. Let $\rho, \rho_{n} \in C_{c}^{\infty}\left(\mathbb{R}^{d}\right)$ be as in Subsection 4.9, then there exists a sequence of convolution operators with $\rho_{n}$

$$
C_{n}: \frac{\Omega_{c}^{\prime 1}(\boldsymbol{\Sigma})}{Z^{d} \Omega_{c}^{\prime 1}(\boldsymbol{\Sigma})} \oplus Z^{\delta} \Omega_{c}^{\prime 1}(\boldsymbol{\Sigma}) \rightarrow \frac{\Omega_{c}^{1}(\boldsymbol{\Sigma})}{Z^{d} \Omega_{c}^{1}(\boldsymbol{\Sigma})} \oplus Z^{\delta} \Omega_{c}^{1}(\boldsymbol{\Sigma})
$$

such that, for any choice of relatively compact open sets $\mathbf{B} \subset \overline{\mathbf{B}} \subset \mathbf{B}_{1} \subset \boldsymbol{\Sigma}$, suppo $\subset \mathbf{B}$ implies supp $C_{n} \alpha \subset \mathbf{B}_{1}$ for large $n$ and $C_{n} \rightarrow \mathbf{1}$ strongly when restricted to $\mathbf{H}(\mathbf{B})$.

Proof. Let $\rho, \rho_{n} \in C_{c}^{\infty}\left(\mathbb{R}^{d}\right)$ be as in Subsection 4.3 consider the convolution operator $C_{\rho_{n}}: \Omega_{c}^{\prime k}(\boldsymbol{\Sigma}) \rightarrow \Omega_{c}^{k}(\boldsymbol{\Sigma})$. It commutes with $d$ and $\delta$, therefore the image of $Z^{d} \Omega^{\prime 1}(\boldsymbol{\Sigma})$ is in $Z^{d} \Omega_{c}^{1}(\boldsymbol{\Sigma})$ and the image of $Z^{\delta} \Omega^{\prime 1}(\boldsymbol{\Sigma})$ is in $Z^{\delta} \Omega_{c}^{1}(\boldsymbol{\Sigma})$, so that $C_{\rho_{n}}$ induces an operator $\widetilde{C_{\rho_{n}}}$ on the quotient space and we define $C_{n}:=\widetilde{C_{\rho_{n}}} \oplus C_{\rho_{n}}$.

In the double cone $O_{1}=C\left(\mathbf{B}_{1}\right)$, the Cauchy surface $\boldsymbol{\Sigma}$ can be identified with $\mathbb{R}^{d}$ and using Fourier transform (see [22]) we prove as in the scalar case that $C_{n} a \rightarrow a$ in $\mathbf{H}(\mathbf{B})$.

Theorem 5.9. For any double cone $O$ such that $\bar{O} \subset M$ with basis $\mathbf{B}$, one-particle space duality 24 is satisfied:

$$
H\left(O^{c}\right)^{c}=H(O), \quad \mathbf{H}(\boldsymbol{\Sigma} \backslash \overline{\mathbf{B}})^{c}=\mathbf{H}(\mathbf{B}) .
$$

Proof. For any $a \in \mathbf{H}(\boldsymbol{\Sigma} \backslash \overline{\mathbf{B}})^{c}$ we want to show that $a \in \mathbf{H}(\mathbf{B})$. The forms $a_{n}:=C_{n} \psi(a)=$ $\left[\mathbf{a}_{n}\right] \oplus \mathbf{e}_{n}$ are in $\frac{\Omega_{c}^{1}(\boldsymbol{\Sigma})}{Z^{d} \Omega_{c}^{1}(\boldsymbol{\Sigma})} \oplus Z^{\delta} \Omega_{c}^{1}(\boldsymbol{\Sigma})$ and for any open set $\mathbf{B}_{1} \supset \overline{\mathbf{B}}$ have support in $\mathbf{B}_{1}$ for large $n$, because $\operatorname{supp}\left(a_{n}\right) \subset \operatorname{supp}(\psi(a))+\operatorname{supp}\left(\rho_{n}\right)$. We have that $\mathbf{e}_{n} \in \Omega^{\prime 1}\left(\mathbf{B}_{1}\right)$ and, by the Remark 5.7 as $\mathbf{B}$ is contractible and has trivial 2-cohomology, also $\mathbf{a}_{n}$ can be chosen in $\Omega^{\prime 1}\left(\mathbf{B}_{1}\right)$, therefore $a_{n} \in \mathbf{H}\left(\mathbf{B}_{\mathbf{1}}\right)$. Finally, $a=\lim a_{n}$ is in $\bigcap_{\mathbf{B}_{\mathbf{1}} \supset \overline{\mathbf{B}}} \mathbf{H}\left(\mathbf{B}_{\mathbf{1}}\right)$ and, by outer regularity and Prop. 5.8 $a \in \mathbf{H}(\mathbf{B})$. 


\subsection{A Special Case of Relative Duality in $V_{+}$}

We are now more specific and consider the forward light cone $V_{+}$and as a Cauchy surface $\boldsymbol{\Sigma}$ the positive time branch of the hyperboloid $x^{2}=c^{2}, c \in \mathbb{R}_{+}$. Let $\mathcal{C} \subset \mathbb{R}^{d}$ be an open cone around a specific (space-like) direction $\mathbf{v} \in \mathbb{R}^{d}$ :

$$
\mathcal{C}=\left\{\mathbf{x} \in \mathbb{R}^{d}:(\mathbf{x}, \mathbf{v})>(1-\epsilon)\|x\|\right\}
$$

with $\mathbf{v}^{2}=1$ and small $\epsilon>0$. Let $\mathbf{A} \subset \boldsymbol{\Sigma}$ be the open set of points obtained by acting on the point $(c, \mathbf{0})$ with the semi-group of boosts with speed belonging to $\mathcal{C}$. $\mathbf{A}$ is an unbounded open set lying on $\boldsymbol{\Sigma}$ with vertex in $(c, \mathbf{0})$ and escaping to light-like infinity along the direction $\mathbf{v}$. This is physically interesting (see Introduction) as a state with an electric charge in $V_{+}$ can be obtained as a limit of neutral states with another compensating charge that is moved to time-like infinity along $\mathbf{A}$. Such a state would be localized in $\mathbf{A}$ or, equivalently, in its causal completion $O:=C(\mathbf{A})$.

We want to prove that relative duality holds for $\mathbf{H}(\mathbf{A}): \mathbf{H}(\boldsymbol{\Sigma} \backslash \overline{\mathbf{A}})^{c}=\mathbf{H}(\mathbf{A})$.

As a first step we use conformal covariance to reduce the problem to a simpler situation in which the ambient space is a double cone. We consider a conformal map $\varphi$ as in Subsection 4.4.2 such that $O_{1}:=\varphi\left(V_{+}\right)$is a double cone with basis $\mathbf{B}_{1}:=\varphi(\boldsymbol{\Sigma})$ lying on the time-0 surface $\mathbb{R}^{d} \subset \mathbb{R}^{d+1}$. B $:=\varphi(\mathbf{A}) \subset \mathbf{B}_{1}$ is a bounded open strongly contractible subset of $\mathbb{R}^{d}$ (the intersection of a cone and $\mathbf{B}_{1}$ ), the causal completion of $\mathbf{B}$ is $C(\mathbf{B})=C(\varphi(\mathbf{A}))=$ $\varphi(C(\mathbf{A}))=\varphi(O)$ and the relative causal complement in $O_{1}$ is $\mathbf{B}^{c}=C(\mathbf{B})^{\prime} \cap O_{1}=\varphi(O)^{\prime} \cap$ $\varphi\left(V_{+}\right)=\varphi\left(O^{\prime} \cap V_{+}\right)=\varphi\left(O^{c}\right)$. As the free electromagnetic field is massless, it is conformally covariant, i.e. there is a unitary implementation $U(\varphi)$ of the conformal map $\varphi$ (cf. 19]): $U(\varphi)^{*} \mathfrak{R}(O) U(\varphi)=\mathfrak{R}(\varphi(O))$ for any $O \subset V_{+}$, therefore $\mathfrak{R}\left(O^{\prime} \cap V_{+}\right)^{\prime} \cap \mathfrak{R}\left(V_{+}\right)=\mathfrak{R}(O)$ $\Longleftrightarrow \mathfrak{R}\left(\varphi(O)^{\prime} \cap \varphi\left(V_{+}\right)\right)^{\prime} \cap \mathfrak{R}\left(\varphi\left(V_{+}\right)\right)=\mathfrak{R}(\varphi(O)) \Longleftrightarrow \mathfrak{R}\left(\mathbf{B}_{1} \backslash \overline{\mathbf{B}}\right)^{c}=\mathfrak{R}(\mathbf{B})$ and again this property can be reduced to the one-particle space duality, namely

$$
\mathbf{H}\left(\mathbf{B}_{1} \backslash \overline{\mathbf{B}}\right)^{c}=\mathbf{H}(\mathbf{B}),
$$

for a strongly contractible subset $\mathbf{B} \subset \mathbf{B}_{1} \subset \mathbb{R}^{d}$.

The one-particle Hilbert space $H_{0}=H\left(\mathbb{R}^{d+1}\right)$, written in terms of Cauchy data on the standard time-0 Cauchy surface, can be described as in 30 and the scalar product in 29 (or the corresponding norm) can be explicitly written in this special case using Fourier transform: $\|[\mathbf{a}] \oplus \mathbf{e}\|^{2}=\left\|P_{T} \mathbf{a}\right\|_{+}^{2}+\|\mathbf{e}\|_{-}^{2}$, where $P_{T}$ is the projection on the transverse (or divergence free) part, $\widehat{P}_{T} a_{i}(\mathbf{p})=\left(\delta_{i, j}-p_{i} p_{j} / \mathbf{p}^{2}\right) \widehat{a}_{j}(\mathbf{p})$, and the norms used are the usual free field norms $\|\mathbf{f}\|_{ \pm}^{2}=\int\|\widehat{\mathbf{f}}(\mathbf{p})\|^{2}\|\mathbf{p}\|^{ \pm 1} d^{d} \mathbf{p}$. There is a one to one correspondence between the vector potential $[\mathbf{a}]$ and the magnetic field $\mathbf{b}=* d \mathbf{a}$. In terms of $\mathbf{b}$ and $\mathbf{e}$ the one particle space norm becomes (cf. [4])

$$
\|\mathbf{b} \oplus \mathbf{e}\|^{2}=\|\mathbf{b}\|_{-}^{2}+\|\mathbf{e}\|_{-}^{2} .
$$

We need a simple lemma which states that there are no elements in the Hilbert space with support on the boundary of a sufficiently regular region.

Lemma 5.10. Let $\mathbf{B}$ be an open bounded strongly contractible set such that $\mathbf{H}(\mathbf{B})$ is outer regular, then there are no non zero elements $a \in H$ such that $\operatorname{supp} \psi(a) \subset \partial \mathbf{B}$.

Proof. Suppose $a \in H$ and $\operatorname{supp} \psi(a) \subset \partial \mathbf{B}$, then by a similar reasoning as in Th. 5.9 above, we can conclude that $a \in \bigcap_{\mathbf{B}_{1} \supset \overline{\mathbf{B}}} \mathbf{H}\left(\mathbf{B}_{\mathbf{1}}\right)$ (notice that $\partial \mathbf{B}$ in general is not contractible but $\mathbf{B}$ is and the sets $\mathbf{B}_{1}$ can be chosen to be). On the other side, $\operatorname{supp} \psi(a) \subset \partial \mathbf{B}$ implies that $\sigma\left(a, a_{1}\right)=0$ for any $a_{1} \in\left[\Omega_{c}^{1}(\mathbf{B})\right] \oplus Z^{\delta} \Omega_{c}^{1}(\mathbf{B})$ and, by continuity of $\sigma$, for any $a_{1} \in \mathbf{H}(\mathbf{B})$, i.e. $a \in \mathbf{H}(\mathbf{B})^{\prime}$. If $\mathbf{H}(\mathbf{B})$ is outer regular, then $a \in \mathbf{H}(\mathbf{B}) \cap \mathbf{H}(\mathbf{B})^{\prime}$ and, as $\sigma$ is non-degenerate on $\mathbf{H}(\mathbf{B}), a=0$. 
In view of Lemma [5.12 which generalizes Lemma [5.10] we prove the following:

Lemma 5.11. For every function $\chi \in C_{c}^{\infty}\left(\mathbb{R}^{d}\right)$, the multiplication operator $M_{\chi}$ on $\Omega_{c}^{1}\left(\mathbb{R}^{d}\right) \oplus$ $\Omega_{c}^{1}\left(\mathbb{R}^{d}\right)$ with the norm 37 is continuous.

Proof. In Fourier transform, the multiplication operator $M_{\chi}$ becomes the convolution operator $T_{\tilde{\chi}}$ with kernel $\tilde{\chi}$ and, according to 37 the norm to be used is $\left\|\omega^{-\frac{1}{2}} \widetilde{\mathbf{b}}\right\|_{2}$, where $\omega$ is the multiplication operator by the function $\omega(\mathbf{p})=\|\mathbf{p}\|$. We have to prove that there exists a constant $C$ such that $\left\|\omega^{-\frac{1}{2}} T_{\widetilde{\chi}} \widetilde{\mathbf{b}}\right\|_{2} \leq C\left\|\omega^{-\frac{1}{2}} \widetilde{\mathbf{b}}\right\|_{2}$ and this follows if we prove that the operator

$$
\omega^{ \pm \frac{1}{2}} T_{\widetilde{\chi}} \omega^{\mp \frac{1}{2}}
$$

is bounded on $L^{2}\left(\mathbb{R}^{d}\right)$. We consider separately the infrared and ultraviolet behavior of the operator. Let $P_{[0,1]}$ and $P_{(1, \infty)}=\mathbf{1}-P_{[0,1]}$ be the spectral operators associated to $\omega(\mathbf{p}) \leq 1$ and $\omega(\mathbf{p})>1$, then we decompose the operator 38 as the sum of $P_{[0,1]} \omega^{ \pm \frac{1}{2}} T_{\tilde{\chi}} \omega^{\mp \frac{1}{2}} P_{[0,1]}$, $P_{[0,1]} \omega^{ \pm \frac{1}{2}} T_{\tilde{\chi} \omega^{\mp \frac{1}{2}}} P_{(1, \infty)}, P_{(1, \infty)} \omega^{ \pm \frac{1}{2}} T_{\widetilde{\chi}} \omega^{\mp \frac{1}{2}} P_{[0,1]}, P_{(1, \infty)} \omega^{ \pm \frac{1}{2}} T_{\tilde{\chi}} \omega^{\mp \frac{1}{2}} P_{(1, \infty)}$ and prove that they are bounded.

First of all, $T_{\tilde{\chi}}$ is bounded by Schur's test: using different estimates for small and for large $\mathbf{p}-\mathbf{q}$, there exist constants $C_{n}$ such that $|\tilde{\chi}(\mathbf{p}-\mathbf{q})| \leq C_{n}\|\mathbf{p}-\mathbf{q}\|^{-n}$, therefore

$$
\begin{aligned}
\sup _{\mathbf{p}} \int|\tilde{\chi}(\mathbf{p}-\mathbf{q})| d \mathbf{q} \leq \sup _{\mathbf{p}}\left\{\int_{\|\mathbf{p}-\mathbf{q}\| \leq 1} C_{0} d \mathbf{q}+\int_{\|\mathbf{p}-\mathbf{q}\|>1} C_{n}\|\mathbf{p}-\mathbf{q}\|^{-n} d \mathbf{q}\right\} \\
=\sup _{\mathbf{p}}\left\{\int_{\|\mathbf{q}\| \leq 1} C_{0} d \mathbf{q}+\int_{\|\mathbf{q}\|>1} C_{n}\|\mathbf{q}\|^{-n} d \mathbf{q}\right\}<+\infty
\end{aligned}
$$

and the same holds when $\mathbf{p}$ and $\mathbf{q}$ are interchanged. Concerning the first three operators, we prove that $\left(\omega^{ \pm \frac{1}{2}} T_{\tilde{\chi}} \omega^{\mp \frac{1}{2}}-T_{\tilde{\chi}}\right) P_{[0,1]}$ is Hilbert-Schmidt, it then follows that $\omega^{ \pm \frac{1}{2}} T_{\tilde{\chi}} \omega^{\mp \frac{1}{2}} P_{[0,1]}$ is bounded and so are its adjoint $P_{[0,1]} \omega^{\mp \frac{1}{2}} T_{\tilde{\chi}} \omega^{ \pm \frac{1}{2}}$ and the three operators. We use the estimate

$$
\left|\frac{\|\mathbf{p}\|^{\frac{1}{2}}-\|\mathbf{q}\|^{\frac{1}{2}}}{\|\mathbf{q}\|^{\frac{1}{2}}}\right|=\left|\frac{\|\mathbf{p}\|-\|\mathbf{q}\|}{\|\mathbf{q}\|^{\frac{1}{2}}\left(\|\mathbf{p}\|^{\frac{1}{2}}+\|\mathbf{q}\|^{\frac{1}{2}}\right)}\right| \leq \frac{\|\mathbf{p}-\mathbf{q}\|}{\|\mathbf{q}\|^{\frac{1}{2}}\|\mathbf{p}\|^{\frac{1}{2}}}
$$

which is a symmetric expression and therefore holds also when $\mathbf{p}$ and $\mathbf{q}$ are interchanged. The kernel of the operator $\left(\omega^{ \pm \frac{1}{2}} T_{\tilde{\chi}} \omega^{\mp \frac{1}{2}}-T_{\tilde{\chi}}\right) P_{[0,1]}$ is square integrable, essentially because the integral of $\tilde{\chi}(\mathbf{p}-\mathbf{q})$ in $\mathbf{q}$ over a compact subset gives a rapid decreasing function of $\mathbf{p}$ :

$$
\begin{gathered}
\iint_{\|\mathbf{q}\| \leq 1}\left|\frac{\|\mathbf{p}\|^{\frac{1}{2}}-\|\mathbf{q}\|^{\frac{1}{2}}}{\|\mathbf{q}\|^{\frac{1}{2}}} \tilde{\chi}(\mathbf{p}, \mathbf{q})\right|^{2} d \mathbf{q} d \mathbf{p} \leq \int\left(\int_{\|\mathbf{q}\| \leq 1}\|\mathbf{p}-\mathbf{q}\|^{2}|\tilde{\chi}(\mathbf{p}-\mathbf{q})|^{2} \frac{d \mathbf{q}}{\|\mathbf{q}\|}\right) \frac{d \mathbf{p}}{\|\mathbf{p}\|} \leq \\
\leq \int_{\|\mathbf{p}\| \leq 2} \int_{\|\mathbf{q}\| \leq 1} C_{1}^{2} \frac{d \mathbf{q}}{\|\mathbf{q}\|} \frac{d \mathbf{p}}{\|\mathbf{p}\|}+\int_{\|\mathbf{p}\|>2}\left(\int_{\|\mathbf{q}\| \leq 1} C_{n+1}^{2}\|\mathbf{p}-\mathbf{q}\|^{-2 n} \frac{d \mathbf{q}}{\|\mathbf{q}\|}\right) \frac{d \mathbf{p}}{\|\mathbf{p}\|}<+\infty
\end{gathered}
$$

The operator is thus Hilbert-Schmidt.

Finally, the operator $P_{(1, \infty)}\left(\omega^{ \pm \frac{1}{2}} T_{\tilde{\chi}} \omega^{\mp \frac{1}{2}}-T_{\tilde{\chi}}\right) P_{(1, \infty)}$ is bounded, again by Schur's test: using the estimate 39] we have that

$$
\begin{aligned}
\sup _{\|\mathbf{p}\| \geq 1} \int_{\|\mathbf{q}\| \geq 1}\left|\frac{\|\mathbf{p}\|^{\frac{1}{2}}-\|\mathbf{q}\|^{\frac{1}{2}}}{\|\mathbf{q}\|^{\frac{1}{2}}} \widetilde{\chi}(\mathbf{p}, \mathbf{q})\right| d \mathbf{q} \leq \sup _{\|\mathbf{p}\| \geq 1} \int_{\|\mathbf{q}\| \geq 1}\|\mathbf{p}-\mathbf{q}\||\widetilde{\chi}(\mathbf{p}-\mathbf{q})| d \mathbf{q} \leq \\
\quad \leq \sup _{\mathbf{p}}\left\{\int_{\|\mathbf{p}-\mathbf{q}\| \leq 1} C_{1} d \mathbf{q}+\int_{\|\mathbf{p}-\mathbf{q}\|>1} C_{n+1}\|\mathbf{p}-\mathbf{q}\|^{-n} d \mathbf{q}\right\}<+\infty
\end{aligned}
$$


and by symmetry the same holds when $\mathbf{p}$ and $\mathbf{q}$ are interchanged.

Lemma 5.12. Let $\mathbf{B}_{1}$ be an open bounded contractible set such that $\mathbf{H}\left(\mathbf{B}_{\mathbf{1}}\right)$ is outer regular, let $a \in \mathbf{H}\left(\mathbf{B}_{\mathbf{1}}\right)$ be such that supp $\psi(a) \subset \overline{\mathbf{B}} \cup \partial \mathbf{B}_{1}$, with $\mathbf{B} \subset \mathbf{B}_{1}$ open set, then supp $\psi(a) \subset \overline{\mathbf{B}}$.

Proof. In order to apply Lemma 5.10 we need to decompose $a$ as a sum of two elements, one with support in $\overline{\mathbf{B}}$ and the other with support in $\partial \mathbf{B}_{1}$. Such a decomposition can be obtained by using a multiplication operator $M_{\chi}$ with a $\chi \in C_{c}^{\infty}\left(\mathbb{R}^{d}\right)$ such that $\chi \equiv 1$ on $\overline{\mathbf{B}}$ and supp $\chi \subset \mathbf{B}_{\epsilon}$, where $\mathbf{B}_{\epsilon}:=\mathbf{B}+\left\{x \in \mathbb{R}^{d}:\|x\|<\epsilon\right\}$. The space $H_{0}$ is a space of divergence free vector fields and is therefore not invariant under multiplication by a function. However, $H_{0}$ is the 'transverse' part of the Hilbert space $K$ defined as the completion of the space of couples of vector fields $\mathbf{e}, \mathbf{b} \in \Omega_{c}^{1}\left(\mathbb{R}^{d}\right)$ with respect to the norm 37 By Prop. [5.6 $\mathbf{e}$ and $\mathbf{b}$ are distributions with support in $\overline{\mathbf{B}} \cup \partial \mathbf{B}_{1}$ belonging to $K$.

By Lemma [5.11] the continuous operator $M_{\chi}$ can be extended from $\Omega_{c}^{1}\left(\mathbb{R}^{d}\right) \oplus \Omega_{c}^{1}\left(\mathbb{R}^{d}\right)$ to the closure $K . M_{\chi} \mathbf{b}$ and $\left(\mathbf{1}-M_{\chi}\right) \mathbf{b}=M_{1-\chi} \mathbf{b}$ are in $K, \operatorname{supp}\left(M_{\chi} \mathbf{b}\right) \subset \operatorname{supp} \chi \cap \operatorname{suppb} \subset \mathbf{B}_{\epsilon}$, $\operatorname{supp}\left(M_{1-\chi} \mathbf{b}\right) \subset \operatorname{supp}(1-\chi) \cap \operatorname{suppb} \subset \partial \mathbf{B}_{1} . M_{1-\chi} \mathbf{b}$ is a distribution with support in $\partial \mathbf{B}_{1}$, but belongs also to a space of free massless vector fields, which are a direct sum of free scalar fields. Therefore, it is outer regular and Lemma 5.10 can be applied to conclude that $M_{1-\chi} \mathbf{b}=0$, thus $\mathbf{b}=M_{\chi} \mathbf{b}$ and $\operatorname{supp}(\mathbf{b}) \subset \mathbf{B}_{\epsilon}$. Similarly for $\mathbf{e}$. It follows that $a$, as a distribution $\psi(a)$, has support in $\mathbf{B}_{\epsilon}$ for $\epsilon$ arbitrary, therefore $\operatorname{supp} \psi(a) \subset \overline{\mathbf{B}}$.

We can now prove the one-particle space duality for the considered regions.

Theorem 5.13. Let $\boldsymbol{\Sigma}=\left\{x \in \mathbb{R}^{d+1}: x_{0} \geq 0, x^{2}=c \in \mathbb{R}_{+}\right\}$be an hyperboloid in the forward light cone $V_{+} \subset \mathbb{R}^{d+1}$ and $\mathcal{C} \subset \mathbb{R}^{d}$ an open cone around a specific (space-like) direction (eq. 35); let $\mathbf{A} \subset \boldsymbol{\Sigma}$ be the open set of points obtained by acting on the point $(c, \mathbf{0})$ with the semigroup of boosts with speed belonging to $\mathcal{C}$ and $C(\mathbf{A}) \subset V_{+}$its causal completion. Then, the isomorphic spaces $H(C(\mathbf{A})) \simeq \mathbf{H}(\mathbf{A})$ satisfy one-particle space duality in $H\left(V_{+}\right) \simeq \mathbf{H}(\boldsymbol{\Sigma})$ :

$$
H\left(C(\mathbf{A})^{c}\right)^{c}=H(C(\mathbf{A})), \quad \mathbf{H}(\boldsymbol{\Sigma} \backslash \overline{\mathbf{A}})^{c}=\mathbf{H}(\mathbf{A}) .
$$

Proof. As explained above, by conformal covariance the previous equation is equivalent to eq. 36] for a strongly contractible subset $\mathbf{B}$ of an open ball $\mathbf{B}_{1} \subset \mathbb{R}^{d}$. Using dilations and the

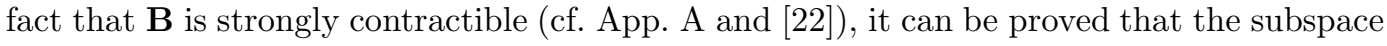
$\mathbf{H}(\mathbf{B})$ is outer regular.

Let $a \in \mathbf{H}\left(\mathbf{B}_{1} \backslash \overline{\mathbf{B}}\right)^{c}$, then $a \in \mathbf{H}\left(\mathbf{B}_{\mathbf{1}}\right)$ implies $\operatorname{supp} \psi(a) \subset \overline{\mathbf{B}_{1}}$ and $a \in \mathbf{H}\left(\mathbf{B}_{\mathbf{1}} \backslash \overline{\mathbf{B}}\right)^{\prime}$ implies $\operatorname{supp} \psi(a) \subset\left(\mathbf{B}_{1} \backslash \overline{\mathbf{B}}\right)^{c}$, hence $\operatorname{supp} \psi(a) \subset \overline{\mathbf{B}} \cup \partial \mathbf{B}_{1}$ and, by Lemma $5.12 \operatorname{supp} \psi(a) \subset$ $\overline{\mathbf{B}}$.

We can now use the same argument as in Th. 5.9 to define $C_{n} \psi(a) \in \frac{\Omega_{c}^{1}\left(\mathbf{B}+\mathbf{B}_{\epsilon}\right)}{Z^{d} \Omega_{c}^{1}(\boldsymbol{\Sigma})} \oplus$ $Z^{\delta} \Omega_{c}^{1}\left(\mathbf{B}+\mathbf{B}_{\epsilon}\right)$ and conclude, using outer regularity of $\mathbf{H}(\mathbf{B})$, that $a=\lim C_{n} a \in \mathbf{H}(\mathbf{B})$.

It then follows the main result of this Subsection:

Theorem 5.14. Let $O:=C(\mathbf{A})$ in the ambient space $V_{+}$as in Th. 5.13, then the algebra of observables for the free electromagnetic field in the representation of the vacuum state satisfies relative duality:

$$
\mathfrak{R}\left(O^{c}\right)^{c}=\mathfrak{R}(O) .
$$

\section{A Outer Regularity}

Outer regularity (Def. 4.5) for $H(O)$ and for $\mathbf{H}(\mathbf{B})$ are equivalent using the isomorphism of eqs. 19 and [20] The first proof of outer regularity for $H(O)$ is in [2] for the massive scalar free field and a class of regions more general than double cones. A simple proof for massive or massless free fields is given in [22] for the following class of regions: 
Definition A.1. A set $\mathbf{B} \subset \mathbb{R}^{d}$ is said strongly contractible around 0 iff for any $\lambda, 0<\lambda<1$, there exists an open neighborhood $\mathbf{A} \subset \mathbb{R}^{d}$ of 0 such that $\lambda(\mathbf{B}+\mathbf{A}) \subset \mathbf{B} . \mathbf{B}$ is said strongly contractible if some of its translated is strongly contractible around 0.

The proof in 22 uses dilation operators: for any $\lambda \in \mathbb{R}_{+}$, the dilation operator $D_{\lambda} f(x):=\lambda^{\frac{d-1}{2}} f(\lambda x)$ can be extended to a bounded operator on $H$ (unitary iff the field is massless) and $D_{\lambda}$ converges strongly to $\mathbf{1}$. Therefore, $f \in \bigcap_{\mathbf{A}} \mathbf{H}(\mathbf{B}+\mathbf{A})$ implies that $D_{\lambda} f \in \mathbf{H}(\mathbf{B})$ for any $\lambda<1$ and $f=\lim _{\lambda \rightarrow 1_{-}} D_{\lambda} f \in \mathbf{H}(\mathbf{B})$.

For the non-contractible regions used in Subsec. 4.4.2 we generalize the idea of 22 using more general diffeomorphisms and prove here the following:

Proposition A.2. Let $O=C(\mathbf{B})$ and $O_{1}=C\left(\mathbf{B}_{1}\right)$ be two double cones with basis $\mathbf{B}$ and $\mathbf{B}_{1}$ on the time-0 Cauchy surface, such that $\bar{O} \subset O_{1}\left(\overline{\mathbf{B}} \subset \mathbf{B}_{1}\right)$; the local space $H\left(O_{1} \cap O^{\prime}\right) \simeq$ $\mathbf{H}\left(\mathbf{B}_{\mathbf{1}} \backslash \overline{\mathbf{B}}\right)$ for the scalar free field (Def. 4.1 ) satisfies outer regularity.

We want to prove that $H\left(O_{1} \cap O^{\prime}\right)=\bigcap_{A} H\left(\left(O_{1} \cap O^{\prime}\right)+A\right)$, where $A$ ranges in the set of open neighborhoods of 0 in $\mathbb{R}^{d+1}$, which is equivalent to

$$
\mathbf{H}\left(\mathbf{B}_{\mathbf{1}} \backslash \overline{\mathbf{B}}\right)=\bigcap_{\mathbf{A}} \mathbf{H}\left(\left(\mathbf{B}_{\mathbf{1}} \backslash \overline{\mathbf{B}}\right)+\mathbf{A}\right),
$$

where $\mathbf{A}$ ranges in the set of open neighborhoods of 0 in $\mathbb{R}^{d}$. As dilations cannot map $\left(\mathbf{B}_{1} \backslash \overline{\mathbf{B}}\right)+\mathbf{A}$ in $\mathbf{B}_{1} \backslash \overline{\mathbf{B}}$, we need more general diffeomorphisms. For $\lambda$ in the neighborhood of 1 , let $\varphi_{\lambda}$ be a family of diffeomorphisms which coincide with the identity outside a fixed compact set. There are well defined constants $a_{\lambda}:=\sup _{\mathbf{x}}\left|\varphi_{\lambda}(\mathbf{x})-\mathbf{x}\right|$ and $b_{\lambda}:=\sup _{\mathbf{x}}\left|\frac{\partial \varphi_{\lambda}}{\partial \mathbf{x}}(\mathbf{x})-\mathbf{1}\right|$ (to simplify the notation $|\cdot|$ indicates the modulus of numbers, the Euclidean norm of vectors or the operator norm of matrices). Suppose that $\varphi_{\lambda}$ converges to the identity as $\lambda \rightarrow 1$, in the sense that $a_{\lambda}, b_{\lambda} \rightarrow 0$.

Proposition A.3. Let $D_{\lambda}$ be the operator on $f_{0} \oplus f_{1} \in C_{c}^{\infty}\left(\mathbb{R}^{d}\right) \otimes \mathbb{R}^{2} \subset \mathbf{H}\left(\mathbb{R}^{d}\right)$ defined by $D_{\lambda} f_{0,1}(x):=f_{0,1}\left(\varphi_{\lambda}(x)\right)$, then $D_{\lambda}$ can be extended to a bounded operator on $\mathbf{H}\left(\mathbb{R}^{d}\right)$. Moreover, $D_{\lambda} \rightarrow \mathbf{1}$ strongly for $\lambda \rightarrow 1$.

Proof. Following Lemma 2.6.1. in [21], for $s<1$ there is a constant $A_{s}$ such that, for any $f \in C_{c}^{\infty}\left(\mathbb{R}^{d}\right)$,

$$
\begin{aligned}
\int \frac{|f(\mathbf{x})-f(\mathbf{y})|^{2}}{|\mathbf{x}-\mathbf{y}|^{d+2 s}} d \mathbf{x} d \mathbf{y}=\int \frac{|f(\mathbf{z}+\mathbf{y})-f(\mathbf{y})|^{2}}{|\mathbf{z}|^{d+2 s}} d \mathbf{z} d \mathbf{y}= \\
=\int \frac{\left|\left(e^{i \mathbf{p z}}-1\right) \widehat{f}(\mathbf{p})\right|^{2}}{|\mathbf{z}|^{d+2 s}} d \mathbf{z} d \mathbf{p}=A_{s} \int|\widehat{f}(\mathbf{p})|^{2}|\mathbf{p}|^{2 s} d \mathbf{p}
\end{aligned}
$$

because for any $\mathbf{z}$ the Fourier transform of the function $\mathbf{y} \mapsto f(\mathbf{z}+\mathbf{y})-f(\mathbf{y})$ is $\mathbf{p} \mapsto$ $\left(e^{i \mathbf{p z}}-1\right) \widehat{f}(\mathbf{p})$ and $\int \frac{\left|\left(e^{i \mathbf{p z}}-1\right)\right|^{2}}{|\mathbf{z}|^{d+2 s}} d \mathbf{z}$ is an homogeneous function of $\mathbf{p}$ of degree $2 s$, thus equal to $A_{s}|\mathbf{p}|^{2 s}$. Therefore, as $\|f\|_{0,1}^{2}=\int|\widehat{f}(\mathbf{p})|^{2}|\mathbf{p}|^{ \pm 1} d \mathbf{p}$, with $\mathbf{x}_{\lambda}^{\prime}:=\varphi_{\lambda}(\mathbf{x})$ we have

$$
\begin{aligned}
\left\|f \circ \varphi_{\lambda}\right\|_{0,1}^{2} & =A_{ \pm}^{-1} \int \frac{\left|f\left(\mathbf{x}_{\lambda}^{\prime}\right)-f\left(\mathbf{y}_{\lambda}^{\prime}\right)\right|^{2}}{|\mathbf{x}-\mathbf{y}|^{d \pm 1}} d \mathbf{x} d \mathbf{y} \leq \\
& \leq A_{ \pm}^{-1}\left(1-b_{\lambda}\right)^{-2 d}\left(1+b_{\lambda}\right)^{d \pm 1} \int \frac{\left|f\left(\mathbf{x}_{\lambda}^{\prime}\right)-f\left(\mathbf{y}_{\lambda}^{\prime}\right)\right|^{2}}{\left|\mathbf{x}_{\lambda}^{\prime}-\mathbf{y}_{\lambda}^{\prime}\right|^{d \pm 1}} d \mathbf{x}_{\lambda}^{\prime} d \mathbf{y}_{\lambda}^{\prime}=\text { const. }\|f\|_{0,1}^{2}
\end{aligned}
$$

where we used the estimate $\sup _{\mathbf{x}}\left|\operatorname{det}\left(\frac{\partial \mathbf{x}}{\partial \mathbf{x}_{\lambda}^{\prime}}\right)\right| \leq \sup _{\mathbf{x}}\left|\frac{\partial \mathbf{x}}{\partial \mathbf{x}_{\lambda}^{\prime}}\right|^{d} \leq\left(1-b_{\lambda}\right)^{-d}$ (from $\frac{\partial \mathbf{x}_{\lambda}^{\prime}}{\partial \mathbf{x}}=$ $\mathbf{1}+\left(\frac{\partial \mathbf{x}_{\lambda}^{\prime}}{\partial \mathbf{x}}-\mathbf{1}\right)$ with $\left|\frac{\partial \mathbf{x}_{\lambda}^{\prime}}{\partial \mathbf{x}}-\mathbf{1}\right| \leq b_{\lambda}<1$, follows the bound for the inverse $\left.\left|\frac{\partial \mathbf{x}}{\partial \mathbf{x}_{\lambda}^{\prime}}\right| \leq \frac{1}{1-b_{\lambda}}\right)$ and 
$\sup _{\mathbf{x}, \mathbf{y}} \frac{\left|\mathbf{x}_{\lambda}^{\prime}-\mathbf{y}_{\lambda}^{\prime}\right|}{|\mathbf{x}-\mathbf{y}|} \leq \sup _{\mathbf{x}}\left|\frac{\partial \mathbf{x}_{\lambda}^{\prime}}{\partial \mathbf{x}}(\mathbf{x})\right| \leq 1+b_{\lambda}$. This proves that the operators $D_{\lambda}$ are bounded, with $\left\|D_{\lambda}\right\|^{2} \leq\left(1-b_{\lambda}\right)^{-2 d}\left(1+b_{\lambda}\right)^{d \pm 1}$.

For a fixed $f$, defining $f_{\lambda}:=f \circ \varphi_{\lambda}-f, \operatorname{supp} f_{\lambda}$ is contained in a fixed compact set for any $\lambda$ and $\left\|f_{\lambda}\right\|_{0,1}^{2}=A_{ \pm}^{-1} \int \frac{\left|f_{\lambda}(\mathbf{x})-f_{\lambda}(\mathbf{y})\right|^{2}}{|\mathbf{x}-\mathbf{y}|^{d \pm 1}} d \mathbf{x} d \mathbf{y} \leq$ const. $\sup _{\mathbf{x}}\left|\frac{\partial f_{\lambda}}{\partial \mathbf{x}}(\mathbf{x})\right|^{2} \rightarrow 0$ because

$$
\begin{array}{r}
\sup _{\mathbf{x}}\left|\frac{\partial f_{\lambda}}{\partial \mathbf{x}}(\mathbf{x})\right|=\sup _{\mathbf{x}}\left|\left(\frac{\partial f}{\partial \mathbf{x}}\left(\mathbf{x}_{\lambda}^{\prime}(\mathbf{x})\right)-\frac{\partial f}{\partial \mathbf{x}}(\mathbf{x})\right) \frac{\partial \mathbf{x}_{\lambda}^{\prime}}{\partial \mathbf{x}}(\mathbf{x})+\frac{\partial f}{\partial \mathbf{x}}(\mathbf{x})\left(\frac{\partial \mathbf{x}_{\lambda}^{\prime}}{\partial \mathbf{x}}(\mathbf{x})-\mathbf{1}\right)\right| \leq \\
\leq \sup _{\mathbf{x}}\left\{\sup _{\mathbf{y}}\left|\frac{\partial^{2} f}{\partial \mathbf{x}^{2}}(\mathbf{y})\right|\left|\mathbf{x}_{\lambda}^{\prime}-\mathbf{x}\right|\left|\frac{\partial \mathbf{x}_{\lambda}^{\prime}}{\partial \mathbf{x}}(\mathbf{x})\right|+\left|\frac{\partial f}{\partial \mathbf{x}}(\mathbf{x})\right|\left|\frac{\partial \mathbf{x}_{\lambda}^{\prime}}{\partial \mathbf{x}}(\mathbf{x})-\mathbf{1}\right|\right\} \leq \\
\leq \sup _{\mathbf{x}}\left|\frac{\partial^{2} f}{\partial \mathbf{x}^{2}}(\mathbf{x})\right| a_{\lambda}\left(1+b_{\lambda}\right)+\sup _{\mathbf{y}}\left|\frac{\partial f}{\partial \mathbf{x}}(\mathbf{y})\right| b_{\lambda} \rightarrow 0
\end{array}
$$

This proves that $\left\|D_{\lambda} f-f\right\| \rightarrow 0$ for $f \in C_{c}^{\infty}\left(\mathbb{R}^{d}\right) \otimes \mathbb{R}^{2}$ and, by the above uniform bound on $\left\|D_{\lambda}\right\|$, for any $f \in \mathbf{H}\left(\mathbb{R}^{d}\right)$.

It is clear that such a family of diffeomorphisms $\varphi_{\lambda}$ can be chosen so that for any $\lambda>1$ there is an open set $\mathbf{A}$ such that $\varphi_{\lambda}\left(\left(\mathbf{B}_{1} \backslash \overline{\mathbf{B}}\right)+\mathbf{A}\right) \subset \mathbf{B}_{1} \backslash \overline{\mathbf{B}}$. As a consequence, $f \in \bigcap_{\mathbf{A}} \mathbf{H}\left(\left(\mathbf{B}_{\mathbf{1}} \backslash \overline{\mathbf{B}}\right)+\mathbf{A}\right)$ implies that, for any $\lambda>1, \varphi_{\lambda}(f) \in \mathbf{H}\left(\mathbf{B}_{\mathbf{1}} \backslash \overline{\mathbf{B}}\right)$ and, taking the limit $\lambda \rightarrow 1, f \in \mathbf{H}\left(\mathbf{B}_{\mathbf{1}} \backslash \overline{\mathbf{B}}\right)$.

\section{B On Differential Forms and the Propagator}

Let $M$ be a globally hyperbolic $d+1$-dimensional manifold and $\boldsymbol{\Sigma}$ any Cauchy (smooth) surface. The Lorentzian metric on $M$ and the induced Riemannian one on $\boldsymbol{\Sigma}$ define canonically a volume form $\omega$ and an inner product on the external algebra of the cotangent space: $a_{p} \cdot b_{p} \in \mathbb{R}$ with $a_{p}, b_{p} \in \Lambda^{*}\left(T_{p}^{*} M\right)$ and $\mathbf{a}_{p} \cdot \mathbf{b}_{p} \in \mathbb{R}$ with $\mathbf{a}_{p}, \mathbf{b}_{p} \in \Lambda^{*}\left(T_{p}^{*} \boldsymbol{\Sigma}\right)$. The inner product on forms with compact support $\Omega_{c}^{k}(M)$ and $\Omega_{c}^{k}(\boldsymbol{\Sigma})$ is the integral of the point-wise inner product $a \cdot b \in \Omega_{c}^{0}(M):\langle a, b\rangle:=-\int(a \cdot b) \omega$. It is indefinite on the first space and positive definite on the second (the minus sign is necessary according to the Lorentzian signature $(+-\ldots-)$ of the metric). The Hodge star operation on differential forms $*: \Omega^{k}(M) \rightarrow \Omega^{d+1-k}(M), *: \Omega^{k}(\boldsymbol{\Sigma}) \rightarrow \Omega^{d-k}(\boldsymbol{\Sigma})$, is defined point-wise according to $\left(a_{p} \cdot b_{p}\right) \omega=a_{p} \wedge * b_{p}$, then $\langle a, b\rangle=-\int a \wedge * b$. On $\Omega^{k}(M), *^{2}=-(-1)^{k d}$ (the extra minus is due to the above signature of the metric) and on $\Omega^{k}(\boldsymbol{\Sigma}), *^{2}=(-1)^{k(d+1)}$.

Through this inner product, $\Omega_{c}^{k}(M)$ is a subset of the space of $k$-currents (set of distributions valued $k$-forms [27] that we denote $\Omega^{\prime k}(M)$ : it is the space of continuous linear functional on $\Omega_{c}^{k}(M)$ with respect to the standard topology as a test forms' space). We indicate again with the same symbol $\langle a, b\rangle$ also the action of the current $a$ on the form $b$.

From the relation $\int a \wedge * b=(-1)^{k(d+1-k)} \int * a \wedge b$, for $a, b \in \Omega_{c}^{k}(M)$, one deduces that the transpose of the exterior differentiation $d: \Omega^{k}(M) \rightarrow \Omega^{k+1}(M)$ is $\delta:=(-1)^{(d+1) k} * d *$ : $\Omega^{\prime k+1}(M) \rightarrow \Omega^{\prime k}(M)$. Similarly $\delta:=(-1)^{d k} * d *$ on $\boldsymbol{\Sigma}$. For simplicity, we use the same letters $d, \delta$ and $*$ for operators on the different spaces $\Omega^{k}(M), \Omega^{k}(\boldsymbol{\Sigma}), \Omega^{\prime k}(M), \Omega^{\prime k}(\boldsymbol{\Sigma})$. The closed elements with respect to $d$ and $\delta$ (cocycles and cycles) are indicated respectively by $Z^{d} \Omega^{k}(M)$ and $Z^{\delta} \Omega^{k}(M)$, the (co)boundary elements respectively by $B^{d} \Omega^{k}(M)$ and $B^{\delta} \Omega^{k}(M)$.

Let $i_{X}: \Omega^{k}(M) \rightarrow \Omega^{k-1}(M)$ be the contraction of a differential form with a vector field $X$; through the metric, we can identify a vector field $X$ with a 1 -form $X^{*}$ and vice versa, such that $\left\langle X^{*} \wedge a, b\right\rangle=\left\langle a, i_{X} b\right\rangle$ for any couple of forms $a$ and $b$, or such that $\left\langle X^{*}, a\right\rangle=a(X)$ for any $a \in \Omega^{1}(M)$. The Lie derivative along a vector field $X$ is $L_{X}=i_{X} d+d i_{X}$.

Given a Cauchy surface $\boldsymbol{\Sigma}$ let $j: \boldsymbol{\Sigma} \hookrightarrow M$ be the injection and $j^{*}: \Omega^{k}(M) \rightarrow$ $\Omega^{k}(\boldsymbol{\Sigma})$ the pull-back of forms. Clearly, $j^{*}$ is the restriction to $\boldsymbol{\Sigma}$ of the tangent component 
of the differential form. The restriction to $\boldsymbol{\Sigma}$ of the normal component of the differential form is $j^{*} i_{X_{n}}$, where $X_{n}$ is any normalized vector field orthogonal to $\boldsymbol{\Sigma}$, and it coincides with $(-1)^{d-k} * j^{*} *: \Omega^{k}(M) \rightarrow \Omega^{k-1}(\boldsymbol{\Sigma})$; it can be verified in a point $p \in \boldsymbol{\Sigma}$ using local coordinates $x^{i}$, which can be chosen to be orthonormal in $p$ and such that $x^{0}=0$ on $\boldsymbol{\Sigma}$.

We define two operators $\tilde{\rho}_{0}, \tilde{\rho}_{1}: \Omega^{k}(M) \rightarrow \Omega^{k}(\boldsymbol{\Sigma}) \oplus \Omega^{k-1}(\boldsymbol{\Sigma})$, extending those already defined on 0 -forms (eq. 5 ): on $\Omega^{k}(M)$

$$
\tilde{\rho}_{0}:=j^{*} \oplus(-1)^{d-k} * j^{*} *, \quad \tilde{\rho}_{1}:=(-1)^{d+1-k}\left(\left[* j^{*} *, d\right] \oplus\left[j^{*}, \delta\right]\right) .
$$

They have two components: the restriction to $\boldsymbol{\Sigma}$ (of the normal derivative) of the tangent and of the normal component of the differential form (the normal derivative on $\boldsymbol{\Sigma}$ can be written as $\left.(-1)^{d+1-k}\left[* j^{*} *, d\right]=j^{*} L_{X_{n}}\right)$. We define also simpler operators that are used in eq. $42 \rho_{0}, \rho_{1}: \Omega^{k}(M) \rightarrow \Omega^{k}(\boldsymbol{\Sigma})$,

$$
\rho_{0}:=j^{*}, \quad \rho_{1}:=(-1)^{d+1-k} * j^{*} * d
$$

and, as in the scalar case, their transpose on the space of currents.

The propagator $E$ and the operator $P$, already defined for the scalar case (see eq. 4 and 111) as convolutions with solutions of the wave equation, can be extended to differential forms with compact support.

Proposition B.1. The following equality (see [6) holds on $\Omega_{c}^{k}(M)$ :

$$
\begin{aligned}
E & =E\left(\tilde{\rho}_{0}^{\prime} \tilde{\rho}_{1}-\tilde{\rho}_{1}^{\prime} \tilde{\rho}_{0}\right) E= \\
& =E\left(\rho_{0}^{\prime} \rho_{1}-\rho_{1}^{\prime} \rho_{0}\right) E+* E\left(\rho_{0}^{\prime} \rho_{1}-\rho_{1}^{\prime} \rho_{0}\right) E * .
\end{aligned}
$$

Proof. Equation 41] is true on $\Omega^{0}(M)$ where it coincides with [6 it can be proven by induction on the degree of the differential forms showing that both sides of eq. 41] commute with $i_{X}$, where $X$ is any constant vector field on $M \subset \mathbb{R}^{d+1}$. As $E$ commutes with $i_{X}$, one has to check that also $\tilde{\rho}_{0}^{\prime} \tilde{\rho}_{1}$ and $\tilde{\rho}_{1}^{\prime} \tilde{\rho}_{0}$ does. One can compute, using local coordinates in the neighborhood of any $p \in \boldsymbol{\Sigma}$, that $\left(j^{*} a\right)_{p} \cdot\left(j^{*} b\right)_{p}+\left(j^{*} * a\right)_{p} \cdot\left(j^{*} * b\right)_{p}=j^{*}(a \cdot b)_{p}$ and thus

$$
\left\langle j^{*} a, j^{*} b\right\rangle_{\Sigma}+\left\langle j^{*} * a, j^{*} * b\right\rangle_{\Sigma}=\int_{\Sigma} * j^{*}(a \cdot b)
$$

Let us indicate with $L_{n}:=L_{X_{n}}$ the Lie derivative along any normalized vector field $X_{n}$ orthogonal to $\boldsymbol{\Sigma}$. $L_{n}$ commutes with $X$ if it is a constant vector field and one can compute

$$
\begin{gathered}
\left\langle a, \tilde{\rho}_{0}^{\prime} \tilde{\rho}_{1} i_{X} b\right\rangle=\left\langle j^{*} a, j^{*} L_{n} i_{X} b\right\rangle_{\Sigma}+\left\langle j^{*} * a, j^{*} L_{n} * i_{X} b\right\rangle_{\Sigma}= \\
=\left\langle j^{*} a, j^{*} i_{X} L_{n} b\right\rangle_{\Sigma}+\left\langle j^{*} * a, j^{*} * i_{X} L_{n} b\right\rangle_{\Sigma}= \\
=\int_{\Sigma} * j^{*}\left(a \cdot i_{X} L_{n} b\right)=\int_{\Sigma} * j^{*}\left(\left(X^{*} \wedge a\right) \cdot\left(L_{n} b\right)\right)= \\
=\left\langle j^{*}\left(X^{*} \wedge a\right), j^{*} L_{n} b\right\rangle_{\Sigma}+\left\langle j^{*} *\left(X^{*} \wedge a\right), j^{*} * L_{n} b\right\rangle_{\Sigma}= \\
=\left\langle\tilde{\rho}_{0}\left(X^{*} \wedge a\right), \tilde{\rho}_{1} b\right\rangle_{\boldsymbol{\Sigma}}=\left\langle X^{*} \wedge a, \tilde{\rho}_{0}^{\prime} \tilde{\rho}_{1} b\right\rangle=\left\langle a, i_{X} \tilde{\rho}_{0}^{\prime} \tilde{\rho}_{1} b\right\rangle .
\end{gathered}
$$

Similarly one checks that $\left\langle a, \tilde{\rho}_{1}^{\prime} \tilde{\rho}_{0} i_{X} b\right\rangle=\left\langle a, i_{X} \tilde{\rho}_{1}^{\prime} \tilde{\rho}_{0} b\right\rangle$.

To prove the second equality [2] we compute

$$
\begin{aligned}
& \left\langle\tilde{\rho}_{0} a, \tilde{\rho}_{1} b\right\rangle_{\Sigma}=\left\langle j^{*} a,\left[* j^{*} *, d\right] b\right\rangle_{\Sigma}-\left\langle * j^{*} * a,\left[j^{*}, \delta\right] b\right\rangle_{\boldsymbol{\Sigma}}= \\
& =\left\langle j^{*} a, * j^{*} * d b\right\rangle_{\boldsymbol{\Sigma}}-\left\langle j^{*} a, d * j^{*} * b\right\rangle_{\boldsymbol{\Sigma}}-\left\langle * j^{*} * a, j^{*} \delta b\right\rangle_{\boldsymbol{\Sigma}}+\left\langle * j^{*} * a, \delta j^{*} b\right\rangle_{\boldsymbol{\Sigma}}= \\
& \quad=\left\langle j^{*} a, * j^{*} * d b\right\rangle_{\boldsymbol{\Sigma}}-\left\langle * j^{*} * a, j^{*} \delta b\right\rangle_{\boldsymbol{\Sigma}}-\left\langle j^{*} a, d * j^{*} * b\right\rangle_{\boldsymbol{\Sigma}}+\left\langle d * j^{*} * a, j^{*} b\right\rangle_{\boldsymbol{\Sigma}}
\end{aligned}
$$


therefore, taking the antisymmetric part in the exchange $0 \leftrightarrow 1$,

$$
\begin{aligned}
& \left\langle\tilde{\rho}_{0} a, \tilde{\rho}_{1} b\right\rangle_{\boldsymbol{\Sigma}}-\left\langle\tilde{\rho}_{0} b, \tilde{\rho}_{1} a\right\rangle_{\boldsymbol{\Sigma}}= \\
& =\left\langle j^{*} a, * j^{*} * d b\right\rangle_{\boldsymbol{\Sigma}}-\left\langle j^{*} b, * j^{*} * d a\right\rangle_{\boldsymbol{\Sigma}}+\left\langle * j^{*} * b, j^{*} \delta a\right\rangle_{\boldsymbol{\Sigma}}-\left\langle * j^{*} * a, j^{*} \delta b\right\rangle_{\boldsymbol{\Sigma}}= \\
& \quad=\left\langle\rho_{0} a, \rho_{1} b\right\rangle_{\boldsymbol{\Sigma}}-\left\langle\rho_{0} b, \rho_{1} a\right\rangle_{\boldsymbol{\Sigma}}+\left\langle\rho_{0} * a, \rho_{1} * b\right\rangle_{\boldsymbol{\Sigma}}-\left\langle\rho_{0} * b, \rho_{1} * a\right\rangle_{\boldsymbol{\Sigma}}
\end{aligned}
$$

and finally $\tilde{\rho}_{0}^{\prime} \tilde{\rho}_{1}-\tilde{\rho}_{1}^{\prime} \tilde{\rho}_{0}=\rho_{0}^{\prime} \rho_{1}-\rho_{1}^{\prime} \rho_{0}+*\left(\rho_{0}^{\prime} \rho_{1}-\rho_{1}^{\prime} \rho_{0}\right) *$.

\section{References}

[1] H. Araki. A lattice of von Neumann algebras associated with the quantum theory of a free Bose field. J. Math. Phys., 4:1343-1362, 1963.

[2] H. Araki. von Neumann algebras of local observables for free scalar field. J. Math. Phys., 5:1-13, 1964.

[3] H. Araki and S. Yamagami. On quasi-equivalence of quasifree states of the canonical commutation relations. Publ. RIMS, Kyoto Univ., 18:283-338, 1982.

[4] G. Benfatto and F. Nicolò. The local von Neumann algebras for the massless scalar free field and the free electromagnetic field. J. Mathematical Phys., 19(3):653-660, 1978.

[5] A. N. Bernal and M. Sánchez. A note on the extendability of compact hypersurfaces to smooth Cauchy hypersurfaces. arXiv:gr-qc/0507018, 2005.

[6] O. Bratteli and D. W. Robinson. Operator Algebras and Quantum Statistical Mechanics. II. Springer-Verlag, Berlin-Heidelberg-New York, 1979.

[7] D. Buchholz. On the structure of local quantum fields with nontrivial interaction. In Proceedings of the International Conference on Operator Algebras, Ideals, and their Applications in Theoretical Physics (Leipzig, 1977), pages 146-153, Leipzig, 1978. Teubner.

[8] D. Buchholz. The physical state space of quantum electrodynamics. Comm. Math. Phys., 85(1):49-71, 1982.

[9] G. Dell'Antonio. Structure of the algebras of some free systems. Commun. Math. Phys., 9:81-117, 1968.

[10] J. Dimock. Algebras of local observables on a manifold. Commun. Math. Phys., 77:219$228,1980$.

[11] J. Dimock. Quantized electromagnetic field on a manifold. Rev. Math. Phys., 4(2):223$233,1992$.

[12] S. Doplicher, R. Haag, and J. E. Roberts. Fields, observables and gauge transformations I. Commun. Math. Phys., 13:1-23, 1969.

[13] S. Doplicher, R. Haag, and J. E. Roberts. Fields, observables and gauge transformations II. Commun. Math. Phys., 15:173-200, 1969.

[14] S. Doplicher, R. Haag, and J. E. Roberts. Local observables and particle statistics I. Commun. Math. Phys., 23:199-230, 1971.

[15] S. Doplicher, R. Haag, and J. E. Roberts. Local observables and particle statistics II. Commun. Math. Phys., 35:49-85, 1974. 
[16] J.-P. Eckmann and K. Osterwalder. An application of Tomita's theory of modular Hilbert algebras: Duality for free Bose fields. J. Funct. Anal., 13:1-12, 1973.

[17] R. Haag. Local Quantum Physics. Texts and Monographs in Physics. Springer-Verlag, Berlin-Heidelberg, 1992.

[18] P. D. Hislop. A simple proof of duality for local algebras in free quantum field theory. J. Math. Phys., 27(10):2542-2550, 1986.

[19] P. D. Hislop. Conformal covariance, modular structure, and duality for local algebras in free massless quantum field theories. Ann. Physics, 185(2):193-230, 1988.

[20] P. D. Hislop and R. Longo. Modular structure of the local algebras associated with the free massless scalar field theory. Commun. Math. Phys., 84(1):71-85, 1982.

[21] L. Hörmander. Linear partial differential operators. Third revised printing. Die Grundlehren der mathematischen Wissenschaften, Band 116. Springer-Verlag New York Inc., New York, 1969.

[22] P. Leyland, J. E. Roberts, and D. Testard. Duality for quantum free fields. unpublished, 1978.

[23] C. Lueders and J. E. Roberts. Local quasiequivalence and adiabatic vacuum states. Commun. Math. Phys., 134:29-63, 1990.

[24] J. Manuceau. $\mathrm{C}^{*}$-algèbre de relations de commutation. Ann. Inst. H. Poincaré, VIII:139-161, 1968.

[25] K. Osterwalder. Duality for free Bose fields. Commun. Math. Phys., 29:1-14, 1973.

[26] P. Sadowski and S. L. Woronowicz. Total sets in quantum field theory. Rep. Mathematical Phys., 2(2):113-120, 1971.

[27] L. Schwartz. Thèorie des Distributions. Paris, Hermann, 1966. 